\title{
Evolutionary branching in deme-structured populations
}

\author{
Joe Yuichiro Wakano ${ }^{\mathrm{a}, \mathrm{b}, *}$, Laurent Lehmann ${ }^{\mathrm{c}}$ \\ a Meiji Institute for Advanced Study of Mathematical Sciences, Meiji University, Tokyo 164-8525, Japan \\ b Japan Science and Technology, PRESTO, Japan \\ c Department of Ecology and Evolution, UNIL Sorge, Le Biophore, 1015 Lausanne, Switzerland
}

\section{A R T I C L E I N F O}

Article history:

Received 11 July 2013

Received in revised form

23 February 2014

Accepted 26 February 2014

Available online 12 March 2014

Keywords:

Inclusive fitness effect on trait variance

Hamilton's rule

Genetic drift

Relatedness

Individual-based simulation

\begin{abstract}
A B S T R A C T
Adaptive dynamics shows that a continuous trait under frequency dependent selection may first converge to a singular point followed by spontaneous transition from a unimodal trait distribution into a bimodal one, which is called "evolutionary branching". Here, we study evolutionary branching in a deme-structured population by constructing a quantitative genetic model for the trait variance dynamics, which allows us to obtain an analytic condition for evolutionary branching. This is first shown to agree with previous conditions for branching expressed in terms of relatedness between interacting individuals within demes and obtained from mutant-resident systems. We then show this branching condition can be markedly simplified when the evolving trait affect fecundity and/or survival, as opposed to affecting population structure, which would occur in the case of the evolution of dispersal. As an application of our model, we evaluate the threshold migration rate below which evolutionary branching cannot occur in a pairwise interaction game. This agrees very well with the individual-based simulation results.
\end{abstract}

(c) 2014 Elsevier Ltd. All rights reserved.

\section{Introduction}

In evolutionary game theory, individuals are allowed to interact with each other and selection will be frequency-dependent. Even in a constant environment, a population may then show intriguing temporal dynamics. For example, if a trait evolves by the accumulation of small mutations and if disruptive selection stemming from frequency-dependent selection is at work, a continuous trait may show convergence to a singular point followed by spontaneous splitting of a unimodal trait distribution into a bimodal (or multimodal) one, referred to as "evolutionary branching" (Metz et al., 1992, 1996; Geritz et al., 1997). Evolutionary branching is predicted to occur at an evolutionarily singular point that is approaching stable (or convergence stable, CS, Eshel, 1983) but not evolutionarily stable (ES), and it is actually observed in individual-based simulations in many models for the evolution of ecological traits (Doebeli et al., 2004; Brännström et al., 2011).

One important contribution of evolutionary game theory and adaptive dynamics is the analytically tractable prediction of the criteria of evolutionary branching, i.e., the CS and non-ES condition (e.g., Eshel, 1983; Geritz et al., 1997), which generally agrees

\footnotetext{
* Corresponding author at: Meiji Institute for Advanced Study of Mathematica Sciences, Meiji University, Tokyo 164-8525, Japan.

E-mail address: joe@meiji.ac.jp (J.Y. Wakano).
}

well with individual-based simulations. This has been applied to a large spectrum of ecological scenarios involving both inter- and intra-specific interactions. However, the standard application of the recipe assumes an infinite and well-mixed population to obtain the stability criteria. Since real populations are always finite and usually have a spatial structure (dispersal is localized and organisms are likely to interact with neighbors), extending the criteria of stability to more realistic models is biologically relevant.

One important contribution of evolutionary game theory and inclusive fitness theory is an analytically tractable measure of selection (or mutant invasion fitness) for deme-structured populations (e.g., Taylor, 1988; Frank, 1998; Rousset, 2004), which provides a condition for convergence stability (Rousset, 2004). Owing to the smallness of local deme size, any analytic measure of selection needs to take into account local fluctuations of allele frequencies induced by genetic drift. This generates positive correlations of mutant frequencies among individuals in the same deme, making mutant-mutant interactions unavoidable. The concept of relatedness plays a crucial role here, as it allows to reduce the problem of computing the full local distribution of mutants (and thus accounting for their interactions) to the simpler problem of computing the probability that two genes sampled from different individuals are identical-by-descent, thereby making tractable the evaluation of invasion fitness. This has been applied to a large number of different social scenarios (e.g., Frank, 1998), and agrees generally well with individual-based simulations (e.g., Bulmer, 1986, Pen, 2000; 
Leturque and Rousset, 2002; Rousset and Ronce, 2004; Guillaume and Perrin, 2006). However, the standard application of the recipe usually ignores the possibility of branching.

Evolution of continuous traits under a wide variety of different biological situations has been studied using adaptive dynamics and inclusive fitness (e.g., Metz et al., 1992; Dieckmann and Law, 1996; Geritz et al., 1997, 1998; Frank, 1998; Rousset, 2004; Wenseleers et al., 2010). Branching condition in structured populations has been studied using the number of successful emigrants descended from a mutant immigrant, $R_{m}$, as invasion fitness measure (e.g., Metz and Gyllenberg, 2001; Parvinen and Metz, 2008; Ajar, 2003). The 2nd-order derivative $R_{m}^{\prime \prime}$ being positive is the non-ES condition in this approach. Day (2001) takes a slightly different approach and calculates the expected fitness of a carrier of the mutant allele under a probability distribution of the number of mutant alleles in the same deme.

It is relevant to mention that the previous approaches compute invasion fitness under the assumption that there are only two types (or alleles), the mutant and the resident, present in the population. From a stochastic process point of view, this is obtained in the asymptotic of rare mutations, where adaptive evolution is described as a monomorphic jump process that gives rise to the so-called canonical equation of adaptive dynamics (Dieckmann and Law, 1996; Champagnat et al., 2006a,b). Strictly speaking, branching is impossible unless at least three alleles segregate in the population (e.g., Wakano and Lehmann, 2012). Thus, the mutant-resident approach based on a two-allele system does not directly deal with evolutionary branching, but only provides an ad-hoc measure of disruptive selection, which matches very well with results from simulations.

There is, however, another approach to describe the adaptive dynamics. This is to model the trait distribution dynamics as in quantitative genetics. Some studies directly deal with the evolution of the full phenotypic distribution (e.g., Sasaki and Ellner, 1995; Jabin and Raoul, 2011; Mirrahimi et al., 2012), while other studies focus only on some important moments of the distribution such as the mean or the variance (Iwasa et al., 1991; Abrams et al., 1993; Day and Taylor, 1996; Sasaki and Dieckmann, 2011). The dynamics of these moments can be derived under some assumptions on the trait distribution, which is called the moment closure. In this distributional context, which is generally applied to panmictic populations, evolutionary branching is characterized by the increase of the variance in the trait distribution (Sasaki and Dieckmann, 2011). This approach can also be extended to finite and well-mixed population models, in which case the trait variance dynamics provides the branching condition in finite populations (Wakano and Iwasa, 2013).

Here, we aim to construct a model to obtain the condition for evolutionary branching from the variance in the trait distribution in a population subdivided into demes of finite size. To that end, we derive moment dynamics to the 2 nd-order of selection and study the 1st (mean) and 2nd (variance) moments. In doing so, we combine elements of inclusive fitness theory, adaptive dynamics, and quantitative genetics to obtain the condition of evolutionary branching in deme structured populations. Using a Gaussian moment closure approximation, the condition for disruptive selection will be expressed analytically. To describe the effect of population structure on selection, we will extend standard coalescence arguments to a quantitative genetics framework.

This paper is organized as follows. We first describe the biological framework of our deme-structured population model and present results of our individual-based simulations as motivating examples. When the migration rate is low, mutant-mutant interactions are more likely to occur and the evolutionary dynamics can be different from that in well-mixed population. By simulation, we first find the threshold migration rate below which evolutionary branching does not occur, illustrating the importance of spatial structure for branching. We then present our mathematical analysis of the condition for evolutionary branching and we finally perform a detailed comparison between simulation results and analytic predictions.

\section{Model and analysis}

\subsection{Main assumptions}

We consider a spatially structured population consisting of $N_{d}$ islands (demes), each of size $N$, thus summing up to $N_{T}=N_{d} N$ adult haploid individuals in total. Each individual $i$ in deme $k$ has a genetically determined continuous trait value $z_{k i}$. Individuals play games and the payoffs determine their fecundity. We assume that a large number of juveniles are produced by each adult, and that a fraction of them disperses randomly to another deme. Adults die with a constant probability and juveniles compete on each deme for the vacated spots so that exactly $N$ individuals in each deme form the next generations of adults. No other exact assumption about social interactions, reproduction, competition, and dispersal is done at this stage (but later for applications). The model can thus take independent demic extinction (or catastrophes) into account so as to capture meta-population processes.

\subsection{A preliminary simulation result}

Before carrying out our derivation of moment dynamics, we present a motivational example satisfying our assumptions and illustrating the role of spatial structure for branching. We run individual-based simulations of a pairwise non-linear public goods game (Doebeli et al., 2004) played within demes under a WrightFisher updating scheme with standard infinite island model of dispersal assumptions, where the migration rate is $m$ (Fig. 1; for simulation details see Section 4). When the migration rate is relatively large $(m=0.6)$, the evolutionary dynamics was similar to that in a well-mixed population, and branching occurred as soon as the trait evolved to reach the convergence stable (CS) value $z^{*}$ (Fig. 1a). For $m=0.4$, branching still occurred but the dynamics was more stochastic (Fig. 1 b). For $m=0.2$, branching was never observed (Fig. 1c). These simulation results clearly illustrate the importance of spatial structure, implying the existence of a threshold migration rate, $m^{*}$, below which evolutionary branching does not occur. One practical goal of our analysis is to give an analytical prediction on this threshold migration rate. We will now derive approximations for the dynamics of the mean and variance in trait value under our population assumptions.

\subsection{Mean trait dynamics to the 1st-order effect of selection}

We write the fitness of individual $i$ in deme $k$ (expected number of adult offspring produced) as a function $w_{k i}(\mathbf{z})$ of the full trait distribution $\mathbf{z}:=\left(z_{11}, z_{12}, \ldots, z_{N_{d} N}\right)$ in the population (see below for examples). The expectation of the mean trait value in the next generation is given by

$E\left[\bar{z}_{t+1} \mid \mathbf{z}_{t}=\mathbf{z}\right]=\frac{1}{N_{T}} \sum_{k=1}^{N_{d}} \sum_{i=1}^{N} z_{k i} w_{k i}(\mathbf{z})$

When the trait distribution is narrow around the mean $\bar{z}:=\left(1 / N_{T}\right) \sum_{k} \sum_{i} z_{k i}$, the deviation $\delta_{k i}:=z_{k i}-\bar{z}$ is small and the fitness function can be approximated by a 1st-order Taylor expansion about the mean:

$w_{k i}(\mathbf{z})=w_{k i}(\overline{\mathbf{z}})+w_{k i, k i} \delta_{k i}+\sum_{j \neq i} w_{k i, k j} \delta_{k j}+\sum_{l \neq k} \sum_{j} w_{k i, l j} \delta_{l j}$ 
where $\overline{\mathbf{z}}:=(\bar{z}, \bar{z}, \ldots, \bar{z})$ and

$w_{k i, l j}:=\frac{\partial w_{k i}(\overline{\mathbf{z}})}{\partial z_{l j}}$

is the partial derivative of the fitness function of an individual $i$ in deme $k$ with respect to the trait value of individual $j$ in deme $l$, evaluated at $\overline{\mathbf{z}}$. Owing to the assumption of constant population size $\bar{w}=1$ and $w_{k i}(\overline{\mathbf{z}})=1$ always hold. Moreover, under the assumption of random dispersal, a derivative of a fitness function depends only on the relationship between two individuals (self, in the same deme, or in different demes). In other words, $w_{k i, l j}$ takes one of three values.

Using this symmetry, putting Eq. (2) into Eq. (1), and rearranging terms (see Appendix A), we have

$E[\Delta \bar{z} \mid \mathbf{z}]=\frac{1}{N_{T}} \sum_{k} \sum_{i}\left(w_{k i, k i} \delta_{k i}^{2}+w_{k i, k j} \delta_{k i} \sum_{j \neq i} \delta_{k j}+w_{k i, j} \delta_{k i} \sum_{l \neq k} \sum_{j} \delta_{l j}\right)$

where $E[\Delta \bar{z} \mid \mathbf{z}]:=E\left[\bar{z}_{t+1}-\bar{z} \mid \mathbf{z}_{t}=\mathbf{z}\right]$. We denote by

$\overline{\delta^{2}}:=\frac{1}{N_{T}} \sum_{k=1}^{N_{d}} \sum_{i=1}^{N}\left(\delta_{k i}\right)^{2}$

the 2nd centralized moment of the trait distribution $\mathbf{z}$, which equals the trait variance $V:=\overline{\delta^{2}}$ in the population. Similarly,

$\overline{\delta \delta}:=\frac{1}{N_{d} N(N-1)} \sum_{k=1}^{N_{d}} \sum_{i=1}^{N} \sum_{j=1, j \neq i}^{N} \delta_{k i} \delta_{k j}$

is the covariance of trait values of pairs of individuals taken in the same deme, and

$\overline{\delta \delta_{d}}:=\frac{1}{N_{d}\left(N_{d}-1\right) N^{2}} \sum_{k=1}^{N_{d}} \sum_{l \neq k i=1}^{N_{d}} \sum_{i=1}^{N} \sum_{j=1}^{N} \delta_{k i} \delta_{l j}$

is the covariance of trait values of pairs of individuals taken in different demes. In terms of these moments, the expected change of the mean trait can be written as

$\begin{aligned} E[\Delta \bar{z} \mid \mathbf{z}] & =w_{S} V+w_{D} \overline{\delta \delta}+w_{O} \overline{\delta \delta_{d}} \\ & =w_{S}\left(V-\overline{\delta \delta_{d}}\right)+w_{D}\left(\overline{\delta \delta}-\overline{\delta \delta_{d}}\right)\end{aligned}$ which follows from the assumed symmetries and

$w_{S}=w_{k i, k i}$

$w_{D}=(N-1) w_{k i, k j} \quad(j \neq i)$

$w_{O}=N\left(N_{d}-1\right) w_{k i, l j} \quad(k \neq l)$

where the right hand side is identical regardless of $i, j, k$ and $l$. These are, respectively, the total fitness effects from Self, Dememates and individuals from Other demes, and $w_{S}+w_{D}+w_{O}=0$ always holds (Rousset, 2004).

When the number of demes is infinite $\left(N_{d} \rightarrow \infty\right)$, the dynamics becomes deterministic and $E[\Delta \bar{z} \mid \mathbf{z}]$ can be replaced by $\Delta \bar{z}$. In addition, the covariance across demes vanishes and the term $\overline{\delta \delta_{d}}$ in Eq. (8) is negligible because $\overline{\delta \delta_{d}}$ is the average of products of the deviations of two independent variables. To derive the moment $\overline{\delta \delta}$, we apply a coalescence argument and write

$\overline{\delta \delta}=R_{2} \overline{\delta^{2}}+\left(1-R_{2}\right)(\bar{\delta})^{2}$

Here, $R_{2}$ is the probability that the ancestral gene lineages sampled from two different individuals in the same deme coalesce in the same ancestor in that deme, in which case the covariance in trait values between the two individuals is $\overline{\delta^{2}}$. With probability $\left(1-R_{2}\right)$ at least one of the ancestral lineages eventually moves out from the deme so that the ancestral lines can be considered as independent (no coalescence occurs), and the covariance between individuals will be zero since $\bar{\delta}=0$. Hence,

$R_{2}=\frac{\overline{\delta \delta}}{V}$

represents the (neutral) relatedness coefficient within demes. Using Eq. (11), in the infinite population limit we obtain the standard formula

$\Delta \bar{z}=V\left[w_{S}+w_{D} R_{2}\right]$

where $w_{S}+w_{D} R_{2}$ is Hamilton's (1964) inclusive fitness effect.

Note that Eq. (12) describes the change in continuous trait value, while the above coalescence argument (Eq. (10)) is usually applied to describe the allele frequency change in a two-allele a
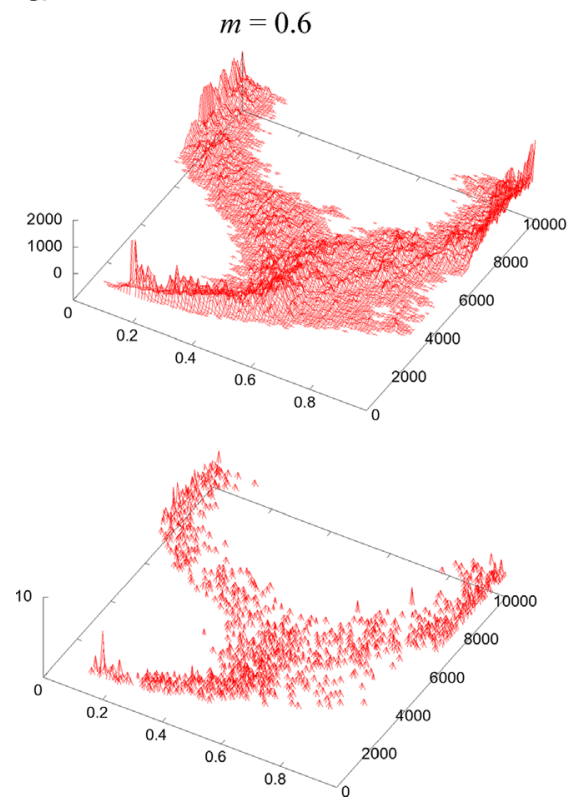

b
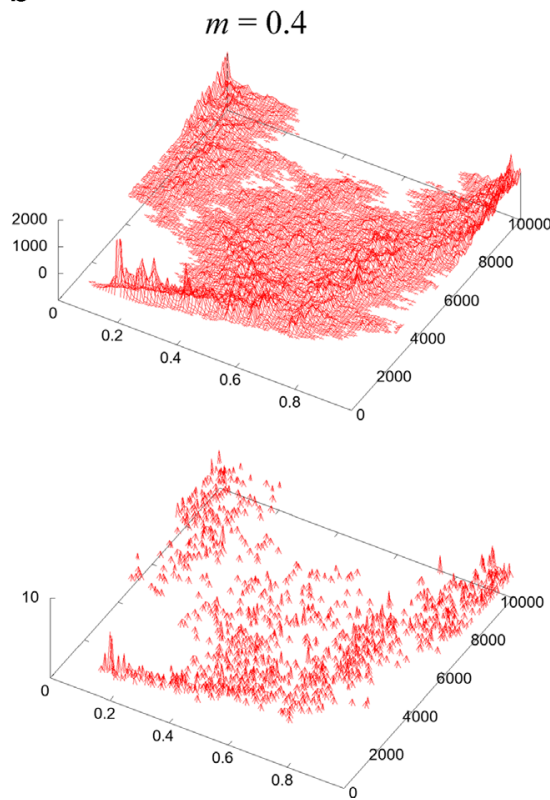

C
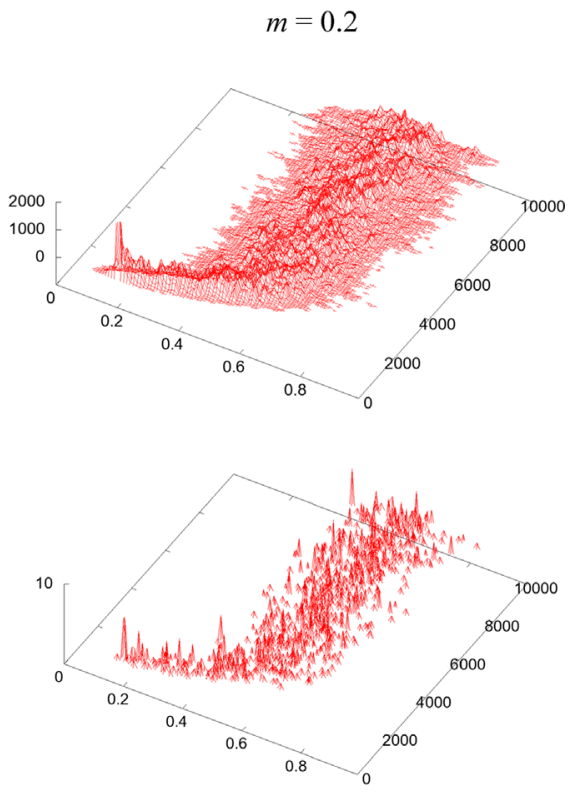

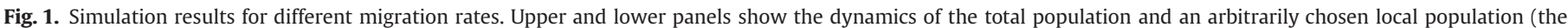
deme with index $k=1)$, respectively. Parameter values are $N_{d}=1000, N=8, \mu=0.01, \sigma=0.02,\left(b_{1}, b_{2}, c_{1}, c_{2}\right)=(6.0,-1.4,4.56,-1.6)$. See Section 4 for details. 
system (mutant-resident system, e.g. Roze and Rousset, 2003; Rousset, 2004). One message behind Eq. (12) is that the inclusive fitness effect for our quantitative genetics approach can be calculated using exactly the same type of coalescence arguments as in the classical setting. Hence, $R_{2}$ under neutrality can be readily calculated for various updating schemes. For instance, for the Wright-Fisher process under the dispersal assumptions of the infinite island model adopted in our simulations (Fig. 1), relatedness is the solution of the classical equation

$R_{2}=(1-m)^{2}\left(\frac{1}{N}+\frac{N-1}{N} R_{2}\right)$

To the 1 st-order effect of selection, an evolutionary singular point $z^{*}$ is given by $w_{S}\left(\mathbf{z}^{*}\right)+w_{D}\left(\mathbf{z}^{*}\right) R_{2}=0$ where $\mathbf{z}^{*}:=\left(z^{*}, z^{*}, \ldots, z^{*}\right)$. If $z^{*}$ satisfies

$Q_{C S}=\left.\frac{d\left(w_{S}(\overline{\mathbf{z}})+w_{D}(\overline{\mathbf{z}}) R_{2}\right)}{d \bar{z}}\right|_{\bar{z}=z^{*}}<0$,

then $z^{*}$ is CS and the mean trait value evolves toward $z^{*}$. This provides exactly the same condition as obtained under the trait substitution sequence assumption (Rousset, 2004).

\subsection{Mean trait dynamics to the 2nd-order effect of selection}

\subsubsection{Taylor expansion of fitness function}

We now apply the same line of reasoning to evaluate the mean trait dynamics to the 2nd-order effect of selection. The Taylor expansion of $w_{k i}(\mathbf{z})$ to the 2 nd-order is given by

$$
\begin{aligned}
& w_{k i}(\mathbf{z})=1+w_{k i, k i} \delta_{k i}+\sum_{j \neq i} w_{k i, k j} \delta_{k j}+\sum_{l \neq k} \sum_{j} w_{k i, j} \delta_{l j} \\
& +\frac{1}{2}\left[\begin{array}{l}
w_{k i, k i k i} \delta_{k i}^{2}+2 \sum_{j \neq i} w_{k i, k i k j} \delta_{k i} \delta_{k j}+2 \sum_{l \neq k} \sum_{j} w_{k i, k i l j} \delta_{k i} \delta_{l j} \\
+\sum_{j \neq i} w_{k i, k j k j} \delta_{k j}^{2}+\sum_{j \neq i} \sum_{n \neq i, j} w_{k i, k j k n} \delta_{k j} \delta_{k n}+2 \sum_{l \neq k} \sum_{j} \sum_{n} w_{k i, k j l} \delta_{k j j} \delta_{l n} \\
+\sum_{l \neq k} \sum_{j} w_{k i, j i j j} \delta_{l j}^{2}+\sum_{l \neq k} \sum_{j} \sum_{n \neq j} w_{k i, j l i} \delta_{l j} \delta_{l n}+\sum_{p \neq k l \neq k} \sum_{j} \sum_{n} w_{k i, j p n} \delta_{l j} \delta_{p n}
\end{array}\right]
\end{aligned}
$$

where

$w_{k i, j p n}:=\frac{\partial w_{k i}(\overline{\mathbf{z}})}{\partial z_{l j} \partial z_{p n}}$

Eq. (15) involves three kinds of 1st-order derivatives and nine kinds of 2nd-order derivatives of a fitness function. Symbols to classify them are shown in Tables 1 and 2. For example, for any $k$ and $i$, we have $w_{S S}:=w_{k i, k i k i}$ which represents the disruptive selection intensity in the case of well-mixed population. Similarly, $w_{S D}:=(N-1) w_{k i, k i k j}$ represents the combined effect from self and another individual in the same deme, $w_{D D}:=(N-1) w_{k i, k j k j}$ represents the 2nd-order effect from a single individual in the same

\section{Table 1}

The 1st-order fitness derivatives and elements of the corresponding moments. "Occurrences" means the number of occurrences in $N_{T}=N_{d} N$ pairs. "Fitness effect" equals the product of "fitness derivative" and "occurrences". "Other" refers to an individual in another island from the focal individual's island. Elements of moments are averaged over population to calculate the moment. Fitness effects are multiplied by the corresponding 2nd-moments (3rd-moments, respectively) in the same row to calculate the mean trait dynamics (the trait variance dynamics, respectively).

\begin{tabular}{llllll}
\hline $\begin{array}{l}\text { Fitness } \\
\text { effect }\end{array}$ & $\begin{array}{l}\text { Fitness } \\
\text { derivative }\end{array}$ & Occurrences Description & $\begin{array}{l}\text { Elements } \\
\text { of 2nd- } \\
\text { moments }\end{array}$ & $\begin{array}{l}\text { Elements } \\
\text { of 3rd- } \\
\text { moments }\end{array}$ \\
\hline$w_{S}$ & $w_{k i, k i}$ & 1 & Self & $\delta_{k i}^{2}$ & $\delta_{k i}^{3}$ \\
$w_{D}$ & $w_{k i, k j}$ & $N-1$ & $\begin{array}{l}\text { Deme- } \\
\text { mate }\end{array}$ & $\delta_{k i} \delta_{k j}$ & $\delta_{k i}^{2} \delta_{k j}$ \\
$w_{O}$ & $w_{k i, l j}$ & $N\left(N_{d}-1\right)$ & Other & $\delta_{k i} \delta_{l j}$ & $\delta_{k i}^{2} \delta_{l j}$ \\
\hline
\end{tabular}

deme and $w_{D D^{\prime}}:=(N-1)(N-2) w_{k i, k j k n}$ represents the combined effect from two different individuals in the same deme.

\subsubsection{Moment closure}

Inserting Eq. (15) to Eq. (1) yields three 2nd-order moments and nine 3rd-order moments, as summarized in Tables 1 and 2 . Each moment is multiplied by the corresponding fitness derivative. The calculation of these moments might result in very complicated expressions when the number of demes is finite, but, as above, we assume that the number of demes is very large $\left(N_{d} \rightarrow \infty\right)$. Then individuals from different demes can be considered as genetically unrelated (their ancestral lineages are independent), which allows us to neglect the moments involving individuals from different demes. Thus, we obtain

$\Delta \bar{z}=w_{S} \overline{\delta^{2}}+w_{D} \overline{\delta \delta}+\frac{1}{2}\left(w_{S S} \overline{\delta^{3}}+\left(2 w_{S D}+w_{D D}\right) \overline{\delta^{2} \delta}+w_{D D^{\prime}} \overline{\delta \delta \delta}\right)$

where we defined

$\overline{\delta^{3}}:=\frac{1}{N_{T}} \sum_{k=1}^{N_{d}} \sum_{i=1}^{N}\left(\delta_{k i}\right)^{3}$,

$\overline{\delta^{2} \delta}:=\frac{1}{N_{d} N(N-1)} \sum_{k=1}^{N_{d}} \sum_{i=1}^{N} \sum_{j=1, j \neq i}^{N}\left(\delta_{k i}\right)^{2} \delta_{k j}$,

and

$\overline{\delta \delta \delta}:=\frac{1}{N_{d} N(N-1)(N-2)} \sum_{k=1}^{N_{d}} \sum_{i=1}^{N} \sum_{j=1, j \neq i l}^{N} \sum_{l=1, l \neq i, l \neq j}^{N} \delta_{k i} \delta_{k j} \delta_{k l}$.

It is sufficient to evaluate these 3rd-order moments appearing in Eq. (17) under neutrality, which we do in two steps. First, we apply the same coalescence argument as above, $\overline{\delta^{i} \delta^{j}}$, whereby

$\overline{\delta^{i} \delta^{j}}=R_{2} \overline{\delta^{i+j}}+\left(1-R_{2}\right) \overline{\delta^{i}} \overline{\delta^{j}}$

Second, we assume that the distribution of phenotypes at a singular point is Gaussian, which is indeed a standard assumption in quantitative genetics and can be derived under the infinite sites model. This leads to the Gaussian closure $\overline{\delta^{3}}=0$ and $\overline{\delta^{4}}=3 V^{2}$ (Kimura, 1965; Sasaki and Dieckmann, 2011, Wakano and Iwasa, 2013). Putting $i=1, j=2, \bar{\delta}=0$ and $\overline{\delta^{3}}=0$ into Eq. (21), we have $\overline{\delta^{2} \delta}=0$. Note that Eq. (21) is only true when selection is absent. We will later calculate the perturbation of $\overline{\delta^{2} \delta}$ in detail.

The moment $\overline{\delta \delta \delta}$ can be thought of as covariance among three different individuals in the same deme. In order to evaluate it, we again use a coalescence argument. The ancestral lineages of the three individuals may all coalesce into a single ancestor, two of them coalesce, or none of them coalesces. So under neutrality we have

$\overline{\delta \delta \delta}=R_{3} \overline{\delta^{3}}+3\left(R_{2}-R_{3}\right) \overline{\delta^{2}} \bar{\delta}+\left(1-R_{3}-3\left(R_{2}-R_{3}\right)\right)(\bar{\delta})^{3}$.

where $R_{3}$ is the probability that the ancestral gene lineage sampled from three different individuals in the same deme coalesce in the same ancestor. This can be thought of as a triplet relatedness, the neutral value of which can again be calculated under different updating schemes. For instance, for Wright-Fisher updating this is a solution of

$R_{3}=(1-m)^{3}\left\{\frac{1}{N^{2}}+3 \frac{N-1}{N^{2}} R_{2}+\left(\frac{N-1}{N}\right)\left(\frac{N-2}{N}\right) R_{3}\right\}$.

Putting $\bar{\delta}=0$ and $\overline{\delta^{3}}=0$ into Eq. (22) yields $\overline{\delta \delta \delta}=0$. Intuitively speaking, it is natural to expect that odd order moments vanish if the phenotypic distribution is symmetric, because odd order products of different variables can take positive or negative values. Thus, Eq. (17) simplifies to

$\Delta \bar{z}=w_{S} \overline{\delta^{2}}+w_{D} \overline{\delta \delta}$ 
Table 2

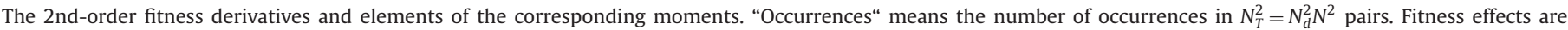

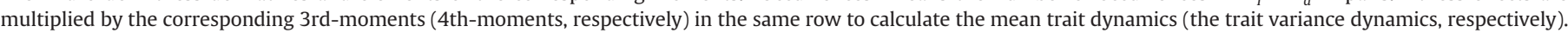

\begin{tabular}{|c|c|c|c|c|c|}
\hline Fitness effects & Fitness derivative & Occurrences & Description & Elements of 3rd-moments & Elements of 4 th-moments \\
\hline$w_{S S}$ & $w_{k i, k i k i}$ & 1 & Self & $\delta_{k i}^{3}$ & $\delta_{k i}^{4}$ \\
\hline$w_{S D}$ & $w_{k i, k i k j}$ & $2(N-1)$ & Self and deme-mate & $\delta_{k i}^{2} \delta_{k j}$ & $\delta_{k i}^{3} \delta_{k j}$ \\
\hline$w_{S O}$ & $w_{k i, k i l j}$ & $2 N\left(N_{d}-1\right)$ & Self and other & $\delta_{k i}^{2} \delta_{l j}$ & $\delta_{k i}^{3} \delta_{l j}$ \\
\hline$w_{D D}$ & $w_{k i, k j k j}$ & $(N-1)$ & Deme-mate & $\delta_{k i} \delta_{k j}^{2}$ & $\delta_{k i}^{2} \delta_{k i}^{2}$ \\
\hline$w_{D D^{\prime}}$ & $w_{k i, k j k p}$ & $(N-1)(N-2)$ & two different deme-mates & $\delta_{k i} \delta_{k j} \delta_{k p}$ & $\delta_{k i}^{2} \delta_{k j} \delta_{k p}$ \\
\hline$w_{D O}$ & $w_{k i, k j l p}$ & $2(N-1)\left(N_{d}-1\right) N$ & Deme-mate and other & $\delta_{k i} \delta_{k j} \delta_{l p}$ & $\delta_{k i}^{2} \delta_{k j} \delta_{l p}$ \\
\hline$w_{O O}$ & $w_{k i, l i j j}$ & $\left(N_{d}-1\right) N$ & Other & $\delta_{k i} \delta_{l j}^{2}$ & $\delta_{k i}^{2} \delta_{l i}^{2}$ \\
\hline$w_{O O^{\prime}}$ & $w_{k i, l j l p}$ & $\left(N_{d}-1\right) N(N-1)$ & Two different others in the same deme & $\delta_{k i} \delta_{l j} \delta_{l p}$ & $\delta_{k i}^{2} \delta_{l j} \delta_{l p}$ \\
\hline$w_{O O_{d}}$ & $w_{k i, l j m p}$ & $\left(N_{d}-1\right)\left(N_{d}-2\right) N^{2}$ & Two different others in different demes & $\delta_{k i} \delta_{l j} \delta_{m p}$ & $\delta_{k i}^{2} \delta_{l j} \delta_{m p}$ \\
\hline
\end{tabular}

In order to evaluate Eq. (24) to the 2nd-order effect of selection, we need to evaluate $\overline{\delta^{2}}$ and $\overline{\delta \delta}$ under selection (this can be thought as the effect of selection on relatedness). However, at a singular point $z^{*}$, where the 1 st-order effect of selection vanishes, the perturbation of these 2 nd-order moments to the 1st-order effect of selection can be expressed only in terms of the 3rd-order moments $\left(\overline{\delta^{3}}, \overline{\delta^{2} \delta}, \overline{\delta \delta \delta}\right)$ evaluated under neutrality. Under our Gaussian closure assumption, these 3rd-order moments vanish. Thus, the 1st-order effect of selection on $\overline{\delta^{2}}$ and $\overline{\delta \delta}$ can be neglected at a singular point $z^{*}$, which entails that the relatedness coefficient must be well approximated by the value estimated under neutrality.

In summary, to the 2nd-order effect of selection, the mean trait dynamics around a singular point can still be approximated by Eq. (12), and this fact will be used in the next section.

\subsection{Trait variance dynamics to the 2nd-order effect of selection}

Since our main goal is to obtain the condition for evolutionary branching, we want to obtain the recursion for the trait variance when $\bar{z}$ has already reached the CS value $z^{*}$. We have shown that the mean trait (i.e., the 1st-moment of trait distribution) does not change even to the 2nd-order effect of selection. It does also not fluctuate as the dynamic is deterministic in infinite populations $\left(N_{d} \rightarrow \infty\right)$. Thus, $\bar{z}_{t+1}=\bar{z}_{t}=z^{*}$ holds. In this situation, we can rescale $z$ to set $\bar{z}=0$ without loss of generality and the expectation of trait variance in the next generation is given by

$E\left[V_{t+1} \mid \mathbf{z}_{t}=\mathbf{z}\right]=\frac{1}{N_{T}} \sum_{k} \sum_{i} \delta_{k i}^{2} w_{k i}(\mathbf{z})$

because the expected number of offspring with trait $z_{k i}=\delta_{k i}$ is $w_{k i}(\mathbf{z})$.

Putting Eq. (15) into Eq. (25) yields three 3rd-order moments and nine 4th-order moments. The terms including 4th-order moments can be calculated in a similar way as in the previous section. On the other hand, the 1st-order effect of selection on 3rdorder moments (i.e., the perturbation of relatedness, see Ajar, 2003) cannot be neglected in general. Thus, Eq. (25) should consist of two terms.

\section{Main result}

\subsection{Condition for evolutionary branching}

In Appendix B, we show that the dynamics of trait variance can be written as

$E[\Delta V \mid \mathbf{z}]=V^{2}(\Delta w+\Delta r)$ where $\Delta w$ quantifies the effect of disruptive selection on the fitness of a focal individual, while $\Delta r$ quantifies that effect through the perturbation of relatedness. Explicitly, we have

$\Delta w=w_{S S}+\left(2 w_{S D}+w_{D D}\right) R_{2}+w_{D D^{\prime}} R_{3}$.

Each term in $\Delta w$ represents a relatedness-weighted 2nd-order effect of various actors on the fitness of a focal recipient. First, $w_{S S}$ stems from the focal individual altering its own trait value. Second, $2 w_{S D}+w_{D D}$ stems from the focal individual and its neighbors altering their trait values $\left(2 w_{S D}\right.$ is the combined effect, while $w_{D D}$ is the effect due solely to neighbors), which is weighted by the association $R_{2}$ between the trait value of the focal individual and any of its neighbors. Third, $w_{D D^{\prime}}$ stems from two distinct neighbors altering their trait values (summed over all possible pairs of neighbors), which is weighted by the association $R_{3}$ between the trait value of the focal individual and the two distinct neighbors.

The general form of the fitness effect $\Delta w$ displayed in Eq. (27) does not depend on the particular demographic assumptions (e.g., Wright-Fisher or Moran). By contrast, $\Delta r$ depends on such assumptions, and for the Wright-Fisher process we have

$\Delta r=4 V^{2} R_{2} \frac{\left(2 R_{2}+(N-2) R_{3}\right) w_{D}^{P}+\left(1+(N-1) R_{2}\right) w_{S}^{P}}{1-m} w_{D}$

where $w_{S}^{P}$ and $w_{D}^{P}$ are fitness derivatives of the philopatric component $w^{P}$ of the fitness function (see Eqs. (B.17), (B.20) and (B.21) in Appendix B).

Note that $\Delta w$ and $\Delta r$ are evaluated at a singular point and thus they are constant. Thus, the trait variance unlimitedly increases if

$Q_{E S}:=\Delta w+\Delta r>0$

Together with the CS condition given by Eq. (14), Eq. (29) can be considered as the branching condition in deme-structured populations.

\subsection{Connection to the previous results}

In the case of a well-mixed population where $R_{2}=R_{3}=0$, Eq. (29) reduces to the traditional non-ES condition (Eshel, 1983); namely,

$w_{S S}=\frac{\partial^{2} w\left(z_{\text {self }}, z_{\text {resident }}\right)}{\partial z_{\text {self }}^{2}}>0$

Most previous studies on structured populations have used the number of successful emigrants descended from a mutant immigrant, $R_{m}$, as invasion fitness measure and studied the branching condition by calculating the 2 nd-order derivative $R^{\prime \prime} m$ (Metz and Gyllenberg, 2001; Ajar, 2003). Nevertheless, Appendix C shows that for the Wright-Fisher process our branching condition reduces to that given in Ajar (2003). The correspondence to the result of Day (2001) is also shown in Appendix C. 
This demonstrates a direct correspondence between the condition of branching obtained by endorsing a mutant-resident system framework and that obtained using a quantitative genetics or distributional framework.

\subsection{Structural-skew effect}

The term $\Delta r$ captures the effect on the trait variance induced by a skew in the trait distribution from the neutral state $\overline{\delta \delta^{2}}=0$. Conceptually, $\Delta r$ measures the 1 st-order perturbation of relatedness due to selection $\left(R_{2}^{\text {selection }}-R_{2}\right)$. It may be expected that such structural-skew effect is limited when population structure is fixed, e.g. constant $m$ and $N$. We will later numerically confirm this $(|\Delta r| \ll|\Delta w|)$ in our example case. As long as branching is mainly induced by disruptive selection intensity ( $\left.w_{S S}\right)$, we can use Eq. (27) as an approximate measure instead of the full branching condition Eq. (29). Note also that for a model where trait changes only fecundity only slightly (weak fecundity selection, see Section 4.3.2), $\Delta r$ can be neglected as it consists of products of 1 st-order fitness derivatives (e.g., $\left.w_{S}^{P} w_{D}\right)$.

\subsection{Trait variance dynamics with mutation}

So far we have only considered the effect of selection. Mutation also increases the trait variance. Genetic drift at the population level is negligible since we assume infinitely many demes. For weak selection and mutation, the variance dynamics can be approximated by

$\frac{d V}{d t}=Q_{E S} V^{2}+\mu \sigma^{2}$

where $\mu$ and $\sigma$ are mutation rate and the standard deviation of mutation step size, respectively (Wakano and Iwasa, 2013). When $Q_{E S}>0$ the dynamics explodes, while when $Q_{E S}<0$ it converges to

$V^{*}=\sqrt{-\frac{\mu \sigma^{2}}{Q_{E S}}}$

The formula gives a quantitative prediction on the effect of migration rate on the trait variance when limited migration suppresses evolutionary branching.

\section{Comparison with simulation results}

\subsection{Pairwise interaction game}

In order to provide an application of our results, we now consider pairwise interactions in each deme. Specifically, every interaction is a two-player game, where the fecundity of individual $i$ in deme $k$ is given by

$F_{k i}=\sum_{j=1, j \neq i}^{N} f\left(z_{k i}, z_{k j}\right) /(N-1)$,

where $f\left(z_{k i}, z_{k j}\right)$ is its payoff when matched with individual $j$ in deme $k$. As an example, we assume that the trait $z$ represents the amount of investment into public goods shared by two players (Doebeli et al., 2004). That is, we have

$f\left(z_{i}, z_{j}\right)=1+B\left(z_{i}+z_{j}\right)-C\left(z_{i}\right)$

where the benefit function

$B(z)=b_{1} z+b_{2} z^{2}$

and the cost function

$C(z)=c_{1} z+c_{2} z^{2}$

are non-linear.
We assume the Wright-Fisher updating. The updating rule in Doebeli et al. (2004) is different from ours, while we faithfully coded the exact Wright-Fisher updating in our simulations to compare with our analytic predictions. The fitness function $w_{k i}$ in terms of fecundity $F_{k i}$ depends on the updating rule. The explicit form is given by Eq. (D.2) in Appendix D.

After reproduction, there is a small probability of mutation $\mu$ per individual, and the mutant trait value follows a normal distribution with a mean equal to the parent's and a variance $\sigma^{2}$. Mutants are constrained to be between 0 and 1 , which confines trait $z$ within an interval $[0,1]$.

\subsection{Evolutionary branching in well-mixed population}

When the population is well-mixed, Doebeli et al. (2004) have shown that the singular strategy $z^{*}=\left(c_{1}-b_{1}\right) /\left(4 b_{2}-2 c_{2}\right)$ is convergence stable (CS) when $2 b_{2}-c_{2}<0$ and evolutionarily stable (ES) when $b_{2}-c_{2}<0$. They have observed evolutionary branching in an individual-based simulation of a large population $(N=10,000)$ when $z^{*}$ is CS but not ES. We confirmed that our calculations of $z^{*}, Q_{C S}$ and $Q_{E S}$ give the same conditions when $R_{2}=R_{3}=0$, and that branching occurred in our simulation with $N_{d}=1$ and $N=8000$ (Fig. 1 in Wakano and Iwasa, 2013).

\subsection{Evolutionary branching in structured populations: analytical results}

\subsubsection{Mean trait dynamics}

When the number of demes is sufficiently large, the inclusive fitness effect in Eq. (12) is given by

$w_{S}+w_{D} R_{2}=\frac{(2-m) m N}{1+(2-m) m(N-1)} s(z)=\left(1-R_{2}\right) s(z)$

where

$s(z)=\frac{b_{1}-c_{1}+\left(4 b_{2}-2 c_{2}\right) z}{1+\left(2 b_{1}-c_{1}+4 b_{2} z-c_{2} z\right) z}$

is the selection gradient in the corresponding well-mixed model. Thus, the singular strategy $z^{*}$ and the CS condition remains the same for deme-structured models and we predict that mean trait dynamics are qualitatively the same in both well-mixed and deme-structured models. Since we adopted the Wright-Fisher updating (no generation overlap), the CS strategy should in effect be identical to that in a well-mixed population (Lehmann, 2008).

\subsubsection{Trait variance dynamics}

The fitness derivatives appearing in our condition for evolutionary branching (Eqs. (27)-(29)) can be explicitly calculated for any pairwise payoff function $f\left(z_{1}, z_{2}\right)$. Thus, we have the branching condition explicitly written in terms of model parameters $\left(b_{1}, b_{2}, c_{1}, c_{2}, m, N\right)$. Although the expression for $Q_{E S}$ becomes cumbersome, the derivation is straightforward and is detailed in Appendix D.

Further, the expression for $Q_{E S}$ can be drastically simplified if one assumes that payoffs affect weakly fitness; namely the cost and benefit in Eqs. (34) are of small order $\varepsilon$ relative to the baseline payoff of one. In this case, one can retain only terms of leading order in $\varepsilon$ in $Q_{E S}$ and all products of fitness derivatives vanish which assures $\Delta r=0$. Then the trait variance unlimitedly increases if

$\frac{\partial^{2} f}{\partial z_{1}^{2}}+\kappa \frac{\partial^{2} f}{\partial z_{2}^{2}}+2(\kappa+\nu) \frac{\partial^{2} f}{\partial z_{1} \partial z_{2}}>0$ 
where

$\kappa=\frac{\left[N-(1-m)^{2}(N-1)\right] R_{2}-(1-m)^{2}}{N-(1-m)^{2}\left[1+(N-1) R_{2}\right]}$

is a (demographically) scaled pairwise relatedness coefficient (Queller, 1994; Lehmann and Rousset, 2010), while

$\nu=\frac{(1-m)^{2}\left[1+(N-3) R_{2}-(N-2) R_{3}\right]}{N-(1-m)^{2}\left[1+(N-1) R_{2}\right]}$
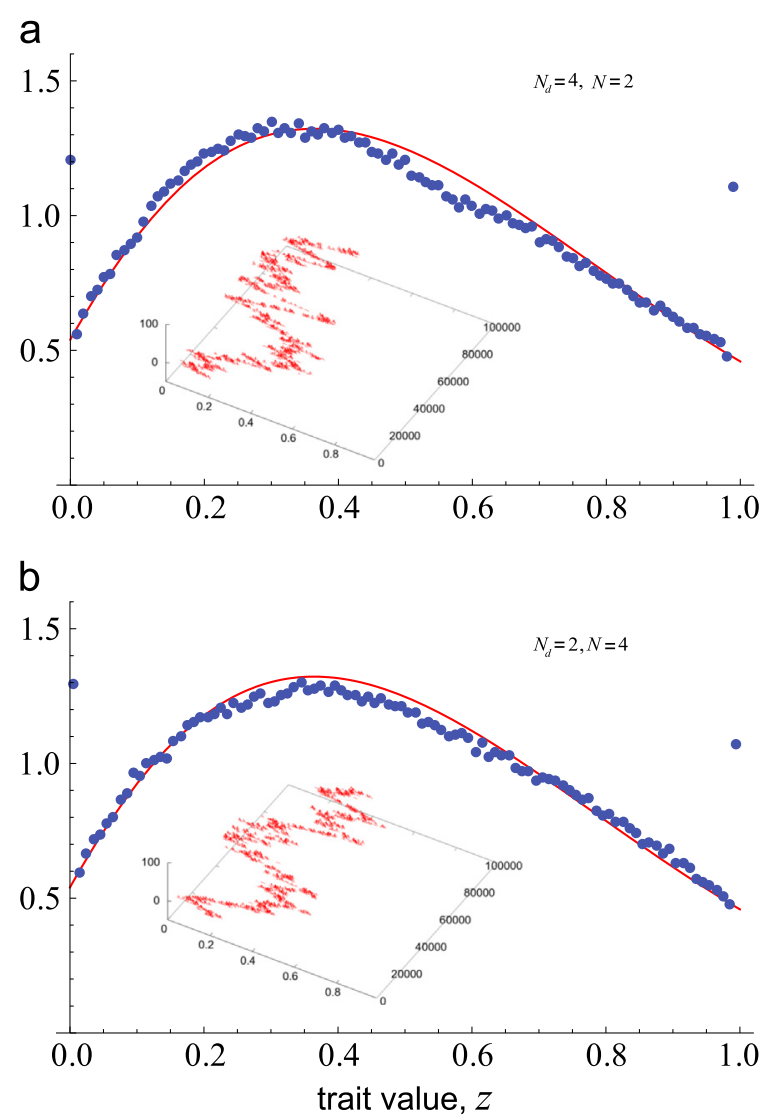

Fig. 2. Results of simulations and analytic predictions for when a singular point is a branching point. Trait distributions averaged over $10^{8}$ generations are shown with inlet panels showing the evolutionary dynamics for the first $10^{5}$ generations. The inlet panels are drawn in the same way as in Fig. 1. Curves represent an analytically obtained distribution $\varphi(z) \sim \int s(z) d z$ where $s(z)$ is the fixation probability perturbation evaluated in a case of finite and well-mixed population (see Wakano and Lehmann, 2012), while circles represent the result of individual-based simulations. Distributions are normalized so that $\int \varphi(z) d z=1$. Simulation results with different migration rates $(m=0.2,0.4,0.6,0.8)$ were almost indistinguishable so only a result with $m=0.2$ is shown. The other parameter values are the same as in Fig. 1. is a (demographically) scaled relatedness coefficient involving triplets of genes. Each updating process will result in a different value for $\kappa$ and $\nu$, and for the Wright-Fisher process we have $\kappa=0$ and

$\nu=\frac{(1-m)^{2}(3-(3-m) m) N-2(1-m)^{4}}{(2-m)(-2+m(3-(3-m) m)(N-2)(N-1)+3 N)}$

The branching condition Eq. (37) is much simpler to compute, since it is expressed directly in terms of payoff and does not require to evaluate any fitness function explicitly. Although Eq. (37) assumes weak fecundity selection, we conjecture that this condition obtains more generally, as long as payoffs affect vital rates (fecundity and survival) by small amount $\varepsilon$ (and this should apply to multiplayer interaction games as well). Thus, $\kappa$ and $\nu$ can be treated as fixed parameters, without the need to explicitly specify a life cycle, with large $\kappa$ and $\nu$ values indicating strong population structure, while small values indicating low population structures.

\subsection{Evolutionary branching in structured populations: simulation} results

\subsubsection{A very small population}

All individual-based simulations were run with fixed game parameters $\left(b_{1}, b_{2}, c_{1}, c_{2}\right)$ for which unique evolutionary branching point $z^{*}=0.6$ exists in a well-mixed model. In order to study the effect of population structure and also to check the correctness of the code, we first investigated very small populations with $N_{T}=8$ individuals divided into either $N_{d}=2$ or 4 demes. In these cases, the evolutionary dynamics should be well-approximated by the trait substitution sequence and evolutionary branching is impossible. The analytic prediction for the stationary distribution $\varphi(z)$ is obtained by the two-allele approximation (Wakano and Lehmann, 2012; Lehmann, 2012), with the selection gradient calculated from the fixation probability perturbation corresponding to the model's assumption (using Eq. (2a) in Eqs. (7) and (8) and Eq. (A.22) of Lehmann, 2008). Our simulation result agreed very well with these theoretical predictions (Fig. 2). As long as the total population size was kept constant, we observed essentially the same result for different structures (e.g., different deme sizes and migration rates).

\subsubsection{A large population}

Next we focused on a population with 1000 demes of size 8 . The population size $N_{\mathrm{T}}=8000$ is sufficiently large to trigger deterministic branching at least in a well-mixed population (Wakano and Iwasa, 2013). Preliminary simulation results (Fig. 1) had already shown the effect of migration rate $m$. The trait variance dynamics for these simulation runs are shown in Fig. 3.
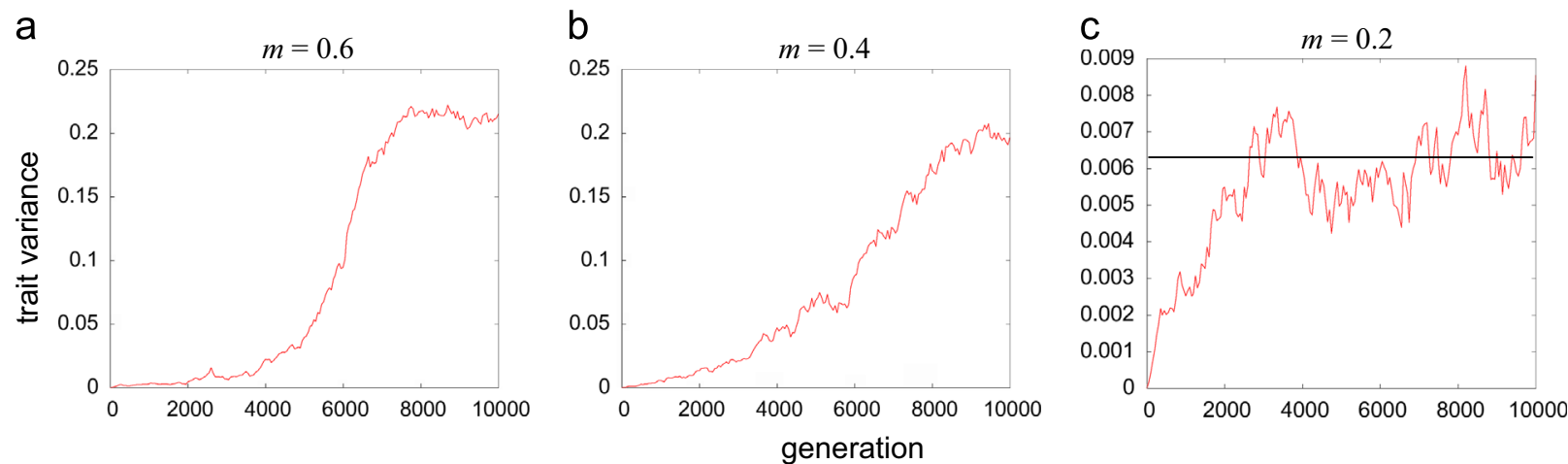

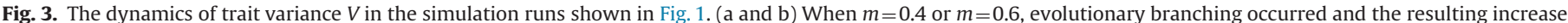
of $V$ was observed. (c) When $m=0.2$, the distribution remained unimodal. Eq. (32) predicts a stable value $V^{*}=0.0063$ (horizontal line). 
For a large migration rate, the trait variance increased sharply at the onset of branching (Fig. 3a). For a small migration rate, the trait variance of a resultant unimodal distribution fluctuated around the equilibrium. This equilibrium value agrees with the predicted value given by Eq. (32) (Fig. 3c).

We substitute parameter values into our branching condition (Eqs. (27)-(29), see also Appendix D) to draw a graph of $Q_{E S}=\Delta w+\Delta r$ as a function of migration rate $m$ (Fig. 4). We numerically confirm that the structural-skew effect $\Delta r$ is negligible. We also find that the simplified condition Eq. (37) is

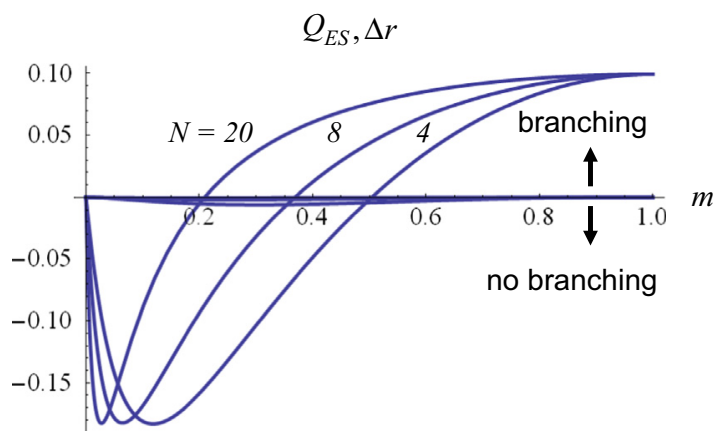

Fig. 4. The dependence of $Q_{E S}=\Delta w+\Delta r$ on migration rate $m$ and deme size $N$ based on analytic result for infinite number of demes (Eqs. (27)-(29)). Both $Q_{E S}$ and $\Delta r$ are drawn showing that $\Delta r$ values are negligibly small compared to $Q_{E S}$. Results for $N=4,8$, and 20 are shown. The threshold migration rates are $m^{*}=0.504$ $0.368,0.210$ for $N=4,8,20$, respectively. Parameter values $\left(b_{1}, b_{2}, c_{1}, c_{2}\right)=$ $(6.0,-1.4,4.56,-1.6)$ are the same as in Fig. 1. numerically very close to the full condition, although game parameters $\left(b_{1}, b_{2}, c_{1}, c_{2}\right)$ are not chosen as very small. Our analysis predicts the existence of the threshold migration rate $m^{*}$ below which branching does not occur. Moreover, it gives a quantitative prediction $m^{*} \approx 0.368$ for $N=8$, which is consistent with our simulation results (Fig. 1). In order to check this prediction more precisely, we have performed simulations for many different $m$ values. Typical dynamics for $m=0.34,0.36$, and 0.38 are shown in Fig. 5. The dynamics looked highly stochastic when $m=0.36$, but they behaved exactly as predicted when $m$ was increased only by 0.02 . As soon as $m$ was increased above $m^{*}$, we observed relatively clear branching dynamics. Several simulations with different combinations of parameter values were performed and they also agreed with our prediction.

\section{Discussion}

Our aim was to derive an analytical condition for evolutionary branching in structured populations using a model of trait distribution dynamics. We have derived the dynamics of the moments (mean and variance) in the total population up to the 2nd-order effect of natural selection. We have shown that the mean trait dynamics can be captured by the traditional inclusive fitness effect. The effect of population structure on the trait variance can then be represented by relatedness coefficients involving two and three individuals evaluated under neutrality. We have derived the condition under which the trait variance explodes. It is the instability condition of a unimodal distribution,
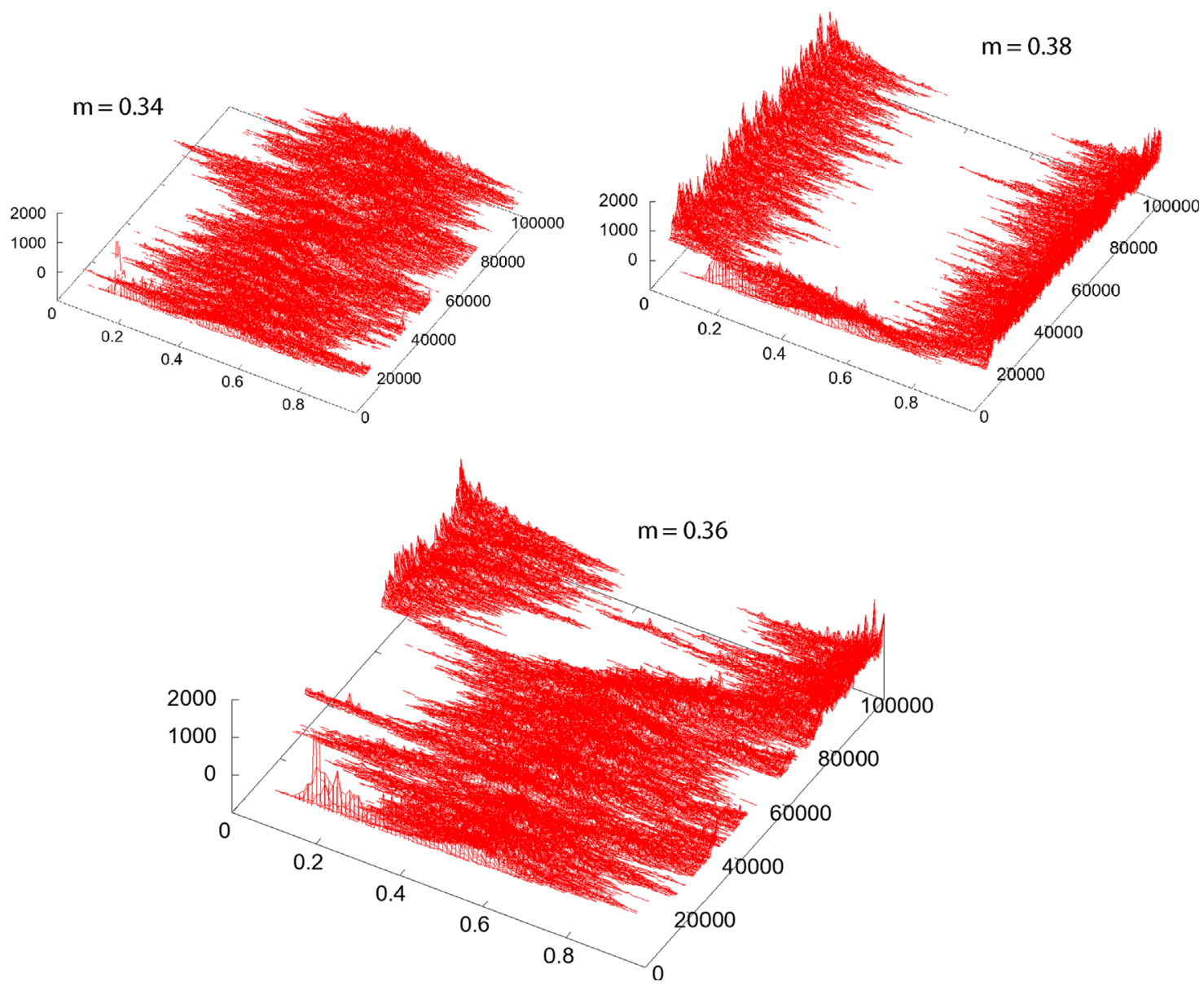

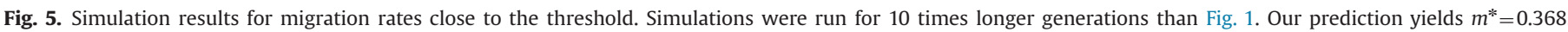

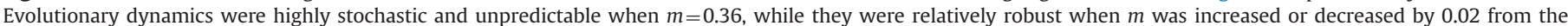
threshold value. The other parameter values are the same as in Fig. 1. 
which parallels the non-ES condition in a mutant-resident system. Together with the CS condition, it can also be considered as the condition of evolutionary branching. Our prediction of branching is qualitatively consistent with individual-based simulations showing that the deme structure suppresses the chance of evolutionary branching in our example (non-linear pairwise public goods game). Moreover, our result is also quantitatively consistent with simulations to predict the value of the minimum migration rate for evolutionary branching to occur.

It is very interesting that we recover the same branching condition as in a previous study that assumed the Wright-Fisher process (Ajar, 2003), although we studied evolutionary branching from a different angle. This confirms the consistency of both approaches; the trait distribution approach (this study) and the mutant-resident approach based on the number of successful mutant emigrants (e.g., Metz and Gyllenberg, 2001; Ajar, 2003; Parvinen and Metz, 2008). One benefit of the trait distribution approach might be that it clarifies the biological interpretation of the condition of disruptive selection $Q_{E S}$. It is the result of selection acting on the trait variance and for example we can predict the variance of a stable unimodal trait distribution (see Eq. (32), Figs. 1 and 3c).

The stability measure $Q_{E S}$ consists of two components. The first component $\Delta w$ includes the disruptive selection intensity that already exists in the corresponding well-mixed model. The second component $\Delta r$ captures the effect of selection on relatedness, which is a form of skew in the trait distribution from what is expected from population structure (e.g., deme size and dispersal rate) when selection is absent. Our numerical calculations have shown that this second component is negligible in a model where population structure is fixed. This is very useful because it circumvents the need to compute $\Delta r$, which is process specific and complicated to obtain, while the expression for $\Delta w$ is general and given by Eq. (27) for all reproductive processes satisfying our demographic assumptions. However, if the evolving trait values affects population structure, such as in a the case of evolution of dispersal rate, the structural-skew effect $\Delta r$, may no longer be negligible (Ajar, 2003).

We further showed that when the evolving trait weakly affects fecundity and/or survival, the condition for branching can be markedly simplified, which allows in this case for a simpler analytical characterization of branching than obtained in previous work (see Sections 3.3 and 4.3.2). Moreover, we observed that this simplified condition still matches well results from simulations even with relatively strong fecundity effects.

We have also focused on a case where the population is subdivided into demes of a constant size. The number of demes was assumed very large to derive analytically tractable equations so that genetic drift at the level of the total population was neglected. Conceptually, these limitations might be relaxed, but calculations may then be difficult for practical purposes. However, preliminary simulations (not presented here) show that the expected waiting time until branching depends on the number of demes in such a way that it agrees with analytic prediction (based on the present study and Wakano and Iwasa, 2013), at least qualitatively.

Our calculation assumes that the trait distribution in the total population obeys the Gaussian distribution, which is not required in the mutant-resident approach. Among the properties of the Gaussian distribution, we only need a Gaussian moment closure that consists of (a) the 3rd moment $\left(\overline{\delta^{3}}\right)$ is negligible and (b) the 4 th moment is three times the variance squared $\left(\overline{\delta^{4}}=3 V^{2}\right)$. The former is quite a reasonable assumption because it holds whenever the trait distribution is symmetric around the mean and because the mean does no longer change once the distribution around the singular point is realized. On the other hand, the latter assumption is more constraining. It can be shown, however, that our result still holds when the kurtosis is not three, but it must be constant. The agreement of the two approaches might imply that the Gaussian distribution assumption is not necessary to justify our results, but clarifying this point is beyond the scope of the present study.

\section{Acknowledgments}

We are grateful to Charles Mullon for various discussions and clarifications. We also thank Hisashi Ohtsuki and Yutaka Kobayashi for valuable comments. This work is partly supported by JSPS KAKENHI No. 25870800 and Swiss NSF grant PP00P3-123344.

\section{Appendix A}

Fitness derivatives satisfy the following equalities for any $i, j, k$, $l, p, q, r$ and $n$ :

$w_{k i, k i}=w_{l j, l j}$

$w_{k i, k j}=w_{k p, k q} \quad$ if $j \neq i$ and $p \neq q$

$w_{k i, l j}=w_{p r, q n} \quad$ if $k \neq l$ and $p \neq q$

Throughout the paper, different indices imply different values. Using this symmetry and putting Eq. (2) into Eq. (1), we have

$$
\begin{aligned}
& E\left[\bar{z}_{t+1} \mid \mathbf{z}_{t}=\mathbf{z}\right] \\
& \quad=\frac{1}{N_{T}} \sum_{k} \sum_{i}\left(\bar{z}+\delta_{k i}\right)\left(1+w_{k i, k i} \delta_{k i}+w_{k i, k j} \sum_{j \neq i} \delta_{k j}+w_{k i, l j} \sum_{l \neq k} \sum_{k j} \delta_{l j}\right)
\end{aligned}
$$

Since the sum of deviations adds up to zero $\left(\sum_{k} \sum_{i} \delta_{k i}=0\right)$, we have

$\sum_{k} \sum_{i} \sum_{j \neq i} \delta_{k j}=\sum_{k} \sum_{i} \sum_{j} \delta_{k j}-\sum_{k} \sum_{i} \delta_{k i}=0$

and

$\sum_{k} \sum_{i} \sum_{l \neq k} \sum_{j} \delta_{l j}=0-\sum_{k} \sum_{i} \sum_{j} \delta_{k j}=0$

which lead to Eq. (4).

\section{Appendix B}

Inserting Eq. (15) into Eq. (25) yields

$E\left[V_{t+1} \mid \mathbf{z}_{t}=\mathbf{z}\right]=w_{S} \overline{\delta^{3}}+w_{D} \overline{\delta \delta^{2}}+V^{2} \Delta w+O\left(\delta^{5}\right)$.

where the first and second terms come from the 1st-order expansion of fitness, while the term $V^{2} \Delta w$ comes from the 2ndorder expansion. We denote by $O\left(\delta^{n}\right)$ the term that only includes the $n$th or higher order products of $\delta_{k i}^{\prime} s$. The derivation of our main result Eqs. (27)-(29) from Eq. (B.1) consists of three steps.

First, we derive the expression of $\Delta w$, i.e., we show the effect of 4th-order moments each of which is associated with a 2nd-order fitness derivative. In contrast to 3rd-order moments, some 4thorder moments among different demes do not vanish because $E\left[X^{2} Y^{2}\right]=E\left[X^{2}\right] E\left[Y^{2}\right]>0$ when $X$ and $Y$ are independent. We have the following non-vanishing 4 th-order moments:

$$
\begin{aligned}
& \overline{\delta^{4}}:=\frac{1}{N_{T}} \sum_{k=1}^{N_{d}} \sum_{i=1}^{N}\left(\delta_{k i}\right)^{4}, \\
& \overline{\delta^{3} \delta}:=\frac{1}{N_{T}(N-1)} \sum_{k=1}^{N_{d}} \sum_{i=1}^{N}\left(\sum_{j=1, j \neq i}^{N}\left(\delta_{k i}\right)^{3} \delta_{k j}\right),
\end{aligned}
$$


$\overline{\delta^{2} \delta^{2}}:=\frac{1}{N_{T}(N-1)} \sum_{k=1}^{N_{d}} \sum_{i=1}^{N}\left(\sum_{j=1, j \neq i}^{N}\left(\delta_{k i}\right)^{2}\left(\delta_{k j}\right)^{2}\right)$,

$\overline{\delta^{2} \delta \delta}:=\frac{1}{N_{T}(N-1)(N-2)} \sum_{k=1}^{N_{d}} \sum_{i=1}^{N}\left(\sum_{j=1, j \neq i n=1, n \neq i, n \neq j}^{N}\left(\delta_{k i}\right)^{2} \delta_{k j} \delta_{k n}\right)$,

$\overline{\delta^{2} \delta_{d}^{2}}:=\frac{1}{N_{T}\left(N_{d}-1\right) N_{k}} \sum_{k=1}^{N_{d}} \sum_{i=1}^{N} \sum_{l=1, l \neq k j=1}^{N_{d}} \sum_{j=1}^{N}\left(\left(\delta_{k i}\right)^{2}\left(\delta_{l j}\right)^{2}\right)$,

and

$\overline{\delta^{2} \delta \delta_{d}}:=\frac{1}{N_{T}\left(N_{d}-1\right) N(N-1)} \sum_{k=1}^{N_{d}} \sum_{i=1}^{N} \sum_{l=1, l \neq k j=1}^{N_{d}} \sum_{n=1, n \neq j}^{N} \sum_{n}^{N}\left(\left(\delta_{k i}\right)^{2} \delta_{l j} \delta_{l n}\right)$.

In terms of these moments which are to be evaluated under neutrality,

$$
\begin{aligned}
V^{2} \Delta w= & \frac{1}{2}\left(w_{S S} \overline{\delta^{4}}+2 w_{S D} \overline{\delta^{3} \delta}+w_{D D} \overline{\delta^{2} \delta^{2}}+w_{D D^{\prime}} \overline{\delta^{2} \delta \delta}+w_{O O} \overline{\delta^{2} \delta_{d}^{2}}\right. \\
& \left.+w_{O O^{\prime}} \overline{\delta^{2} \delta \delta_{d}}\right)
\end{aligned}
$$

holds where we defined $w_{O O}=\left(N_{d}-1\right) N w_{k i, j l j}$ and $w_{O O^{\prime}}=$ $\left(N_{d}-1\right) N(N-1) w_{k i, j l i n}$. Using Eq. (21) and also $\overline{\delta^{2} \delta_{d}^{2}}=V^{2}$ and $\overline{\delta^{2} \delta \delta_{d}}=V \overline{\delta \delta}=R_{2} V^{2}$, we have

$\Delta w=\frac{1}{2}\left(3 w_{S S}+6 w_{S D} R_{2}+w_{D D}\left(1+2 R_{2}\right)+w_{O O}+w_{O O^{\prime}} R_{2}\right)+w_{D^{\prime} D^{\prime}} \overline{\delta^{2} \delta \delta}$

The moment $\overline{\delta^{2} \delta \delta}$ can be evaluated by a similar coalescence argument that we used to derive Eq. (22). This yields

$$
\begin{aligned}
\overline{\delta^{2} \delta \delta}= & R_{3} \overline{\delta^{4}}+2\left(R_{2}-R_{3}\right) \overline{\delta^{3}} \bar{\delta}+\left(R_{2}-R_{3}\right) \overline{\delta^{2}} \overline{\delta^{2}} \\
& +\left(1-R_{3}-3\left(R_{2}-R_{3}\right)\right) \overline{\delta^{2}} \bar{\delta} \bar{\delta}
\end{aligned}
$$

and we have

$\Delta w=\frac{1}{2}\left(3 w_{S S}+w_{D D}+w_{O O}+\left(6 w_{S D}+2 w_{D D}+w_{D^{\prime} D}+w_{O O^{\prime}}\right) R_{2}+2 w_{D^{\prime} D} R_{3}\right)$

To simplify this, we use the fact

$\sum_{k=1}^{N_{d}} \sum_{i=1}^{N} w_{k i}=N_{T}$

By differentiating this equality, we have

$\frac{\partial^{2}}{\partial z_{l j}^{2}}\left(\sum_{k=1}^{N_{d}} \sum_{i=1}^{N} w_{k i}\right)=\sum_{k=1}^{N_{d}} \sum_{i=1}^{N} \frac{\partial^{2} w_{k i}}{\partial z_{l j}^{2}}=w_{S S}+w_{D D}+w_{O O}=0$

and

$(N-1) \frac{\partial^{2}}{\partial z_{l i} \partial z_{l j}}\left(\sum_{k=1}^{N_{d}} \sum_{i=1}^{N} w_{k i}\right)=2 w_{S D}+w_{D D^{\prime}}+w_{O O^{\prime}}=0$

Using these two equalities, we obtain a simplified expression

$\Delta w=w_{S S}+\left(2 w_{S D}+w_{D D}\right) R_{2}+w_{D D^{\prime}} R_{3}$

which finishes the first part.

We now calculate the perturbation of the first 3rd-order moments appearing in Eq. (B.1). After selection, this 3rd-order moment in the next generation is given by

$E\left[\overline{\delta^{3}}{ }_{t+1} \mid \mathbf{z}_{t}=\mathbf{z}\right]:=\frac{1}{N_{T}} \sum_{k} \sum_{i} \delta_{k i}^{3} w_{k i}(\mathbf{z})=\overline{\delta^{3}}+w_{S} \overline{\delta^{4}}+w_{D} \overline{\delta \delta^{3}}+O\left(\delta^{5}\right)$.
Since 4th-order moments are evaluated under neutrality at singular point, we have

$w_{S} \overline{\delta^{4}}+w_{D} \overline{\delta \delta^{3}}=\left(w_{S}+R_{2} w_{D}\right) \overline{\delta^{4}}=0$.

Thus, if $\overline{\delta^{3}}=0$ then it remains zero (precisely, $O\left(\delta^{5}\right)$ ) even after selection. This means that the perturbation of the term $w_{S} \overline{\delta^{3}}$ in Eq. (B.1) can be neglected, which is consistent with the Gaussian closure.

Finally, we now compute the dynamics of the moment $\overline{\delta \delta^{2}}$ in Eq. (B.1) for a Wright-Fisher process which will turn out to be complicated even though we assume Wright's island model. To calculate this, we split fitness into phillopatric and dispersal (allopatric) components:

$w_{k i}=w_{k i}^{P}+\sum_{h \neq k} w_{h k i}^{D}$

where $w_{h k i}^{D}$ is the expected number of adult offspring in deme $h$ of individual $i$ in deme $k$. An individual in deme $k$ descends from an individual $i$ in deme $k$ with probability $w_{k i}^{P} / N$. Two individuals (say, $\mathrm{A}$ and $\mathrm{B}$ ) in deme $k$ descend from the individual $i$ in deme $k$ with probability $\left(w_{k i}^{P} / N\right)^{2}$, and they descend from individuals $i$ and $j$ $(\neq i)$ in deme $k$ with probability $\left(w_{k i}^{P} / N\right)\left(w_{k j}^{P} / N\right)$. We can then write

$E\left[\overline{\delta \delta^{2}}{ }_{t+1} \mid \mathbf{z}_{t}=\mathbf{z}\right]=\varphi+\phi$

where

$\varphi=\frac{1}{N_{d}} \sum_{k}\left(\sum_{i}\left(\frac{w_{k i}^{P}(\mathbf{z})}{N}\right)^{2} \delta_{k i}^{3}+\sum_{i} \sum_{j \neq i} \frac{w_{k i}^{P}(\mathbf{z}) w_{k j}^{P}(\mathbf{z})}{N^{2}} \delta_{k i}^{2} \delta_{k j}\right)$

represents the case when both individuals in deme $k$ (i.e., A and B) descended from deme $k$. The term $\varphi$ takes into account effect due to allopatry and will be calculated explicitly later. Putting the last term in the following Taylor expansion into Eq. (B.19):

$w_{k i}^{P}=(1-m)+w_{k i, k i}^{P} \delta_{k i}+\sum_{j \neq i} w_{k i, k j}^{P} \delta_{k j}+\sum_{h \neq k} \sum_{j} w_{k i, h j}^{P} \delta_{h j}+O\left(\delta^{2}\right)$.

produces moments that will be zero at neutrality. Thus, we can approximate this full expression by

$w_{k i}^{P}=(1-m)+w_{S}^{P} \delta_{k i}+w_{D}^{P} \frac{1}{N-1} \sum_{j \neq i} \delta_{k j}+O\left(\delta^{2}\right)$,

where $w_{S}^{P}:=w_{k i, k i}^{P}$ and $w_{D}^{P}:=(N-1) w_{k i, k j}^{P}$, whereby

$\left(w_{k i}^{P}\right)^{2}=(1-m)^{2}+2(1-m)\left[w_{S}^{P} \delta_{k i}+w_{D}^{P} \frac{1}{N-1} \sum_{j \neq i} \delta_{k j}\right]+O\left(\delta^{2}\right)$,

which yields several 4th-order moments evaluated under neutrality. The first term in $\varphi$ is given by

$\frac{1}{N_{d}} \sum_{k}\left(\sum_{i} \delta_{k i}^{3}\left(\frac{w_{k i}^{P}(\mathbf{z})}{N}\right)^{2}\right)=\frac{(1-m)}{N}\left(2 w_{S}^{P} \overline{\delta^{4}}+2 w_{D}^{P} \overline{\delta \delta^{3}}\right)$.

and the second term in $\varphi$ is given by (after some calculations)

$\frac{1}{N_{d}} \sum_{k} \sum_{i} \sum_{j \neq i} \frac{w_{k i}^{P}(\mathbf{z}) w_{k j}^{P}(\mathbf{z})}{N^{2}} \delta_{k i}^{2} \delta_{k j}=(1-m)^{2} \frac{N-1}{N} \overline{\delta \delta^{2}}+X+O\left(\delta^{5}\right)$

where $X$ is of $O\left(\delta^{4}\right)$ and given by

$$
\begin{aligned}
X & =\frac{1-m}{N^{2} N_{d}} \sum_{k} \sum_{i} \sum_{j \neq i}\left(w_{S}^{P} \delta_{k i}+w_{D}^{P} \overline{1}-1\right. \\
& \left.\sum_{l \neq i} \delta_{k l}+w_{S}^{P} \delta_{k j}+w_{D}^{P} \overline{N-1} \sum_{l \neq j} \delta_{k l}\right) \delta_{k i}^{2} \delta_{k j} \\
& \left.=\frac{(1-m)}{N}\left((N-1) w_{S}^{P}\left(\overline{\delta \delta^{3}}+\overline{\delta^{2} \delta^{2}}\right)+w_{D}^{P} \overline{\left(\delta^{2} \delta^{2}\right.}+\overline{\delta \delta^{3}}+2(N-2) \overline{\delta \delta \delta^{2}}\right)\right)
\end{aligned}
$$

The sum of Eq. (B.23) and (B.24) then gives

$\varphi=(1-m)^{2} \frac{N-1}{N} \overline{\delta \delta^{2}}$ 


$$
\begin{aligned}
& +\frac{(1-m)}{N}\left(w_{S}^{P}\left(2 \overline{\delta^{4}}+(N-1) \overline{\left(\delta \delta^{3}\right.}+\overline{\delta^{2} \delta^{2}}\right)\right) \\
& \left.\left.+w_{D}^{P} \overline{\left(\delta^{2} \delta^{2}\right.}+3 \overline{\delta \delta^{3}}+2(N-2) \overline{\delta \delta \delta^{2}}\right)\right)
\end{aligned}
$$

The term $\phi$ in Eq. (B.18) comes from the case when two individuals sampled in a deme descend from different demes through dispersal. Since the population is of constant size, $w_{h k i}^{D}$ is also $N$ times the probability that an individual sampled in $h$ descends from individual $i$ in deme $k$. We have

$\phi=\frac{1}{N_{d}} \sum_{k}\left(\begin{array}{l}\sum_{i} \sum_{h \neq k} \sum_{j} \frac{w_{k i}^{p} w_{k h j}^{D}}{N^{2}}\left(\delta_{k i}^{2} \delta_{h j}+\delta_{k i} \delta_{h j}^{2}\right) \\ +\sum_{h \neq k} \sum_{j} \sum_{l \neq k} \sum_{i} \frac{w_{k h j}^{D} w_{k l i}^{D}}{N^{2}} \delta_{h j}^{2} \delta_{l i}\end{array}\right)$

The Taylor expansion of $w_{h k j}^{D}$ is

$$
\begin{aligned}
w_{k h j}^{D}= & \frac{m}{N_{d}-1}+\sum_{i} w_{k h j, k i}^{D} \delta_{k i}+w_{k h j, h j}^{D} \delta_{h j}+\sum_{n \neq j} w_{k h j, h n}^{D} \delta_{h n} \\
& +\sum_{l \neq k, h} \sum_{i} w_{k h j, l i}^{D} \delta_{l i}+O\left(\delta^{2}\right),
\end{aligned}
$$

The second term equals to $w_{k h j, k i}^{D} \delta_{k}$ where $\delta_{k}:=\sum_{i} \delta_{k i}$. The last term produces moments for individuals taken in three different demes, which vanish under neutrality. Thus, we can approximate the full expression by

$w_{k h j}^{D}=\frac{1}{N_{d}-1}\left[m+w_{C}^{D} \frac{1}{N} \delta_{k}+w_{S}^{D} \delta_{h j}+w_{D}^{D} \frac{1}{N-1} \sum_{n \neq j} \delta_{h n}\right]$.

where we defined

$w_{S}^{D}:=\sum_{k \neq h} w_{k h j, h j}^{D}=\left(N_{d}-1\right) w_{k h j, h j}^{D}$

$w_{D}^{D}:=\sum_{k \neq h n \neq j} \sum_{k h j, h n} w_{d}^{D}\left(N_{d}-1\right)(N-1) w_{k h j, h n}^{D}$

$w_{C}^{D}:=\sum_{k \neq h} \sum_{i} w_{k h j, k i}^{D}=\left(N_{d}-1\right) N w_{k h j, k i}^{D}$

Note that $w_{C}^{D}$ represents the effect of individuals changing their trait values in a target deme on the dispersing component of fitness. Each summation in Eq. (B.27) becomes a product and we have

$\phi=\frac{1}{N_{d}}\left(\begin{array}{l}\left.\frac{1}{N^{2}}\left\{\sum_{i} w_{k i}^{P} \delta_{k i}^{2}\right\}\left\{\sum_{h \neq k} \sum_{j} w_{k h j}^{D} \delta_{h j}\right\}+\frac{1}{N^{2}}\left\{\sum_{i} w_{k i}^{P} \delta_{k i}\right\}\left\{\sum_{h \neq k} \sum_{j} w_{k h j}^{D} \delta_{h j}^{2}\right\}\right) \\ +\frac{1}{N^{2}}\left\{\sum_{h \neq k} \sum_{j} w_{k h j}^{D} \delta_{h j}^{2}\right\}\left\{\sum_{l \neq k} \sum_{i} w_{k l i}^{D} \delta_{l i}\right\}\end{array}\right)$

$$
=\phi_{1}+\phi_{2}+\phi_{3} \text {. }
$$

Using Eq. (B.29) and taking the limit $N_{d} \rightarrow \infty$, the leading terms are given by

$$
\begin{aligned}
& \sum_{h \neq k} \sum_{j} w_{k h j}^{D} \delta_{h j}=\frac{m}{N_{d}-1} \sum_{h \neq k} \sum_{j} \delta_{h j}=\frac{m}{N_{d}-1}\left(-\delta_{k}\right) \rightarrow 0 \\
& \sum_{h \neq k} \sum_{j} w_{k h j}^{D} \delta_{h j}^{2} \rightarrow m N \overline{\delta^{2}}
\end{aligned}
$$

and we have

$$
\begin{aligned}
& \left\{\sum_{i} w_{k i}^{P} \delta_{k i}^{2}\right\}\left\{\sum_{h \neq k} \sum_{j} w_{k h j}^{D} \delta_{h j}\right\} \\
& =\left\{\sum_{i}\left[(1-m) \delta_{k i}^{2}+w_{S}^{P} \delta_{k i}^{3}+w_{D}^{P} \frac{1}{N-1} \sum_{j \neq i} \delta_{k j} \delta_{k i}^{2}\right]\right\} \\
& \left\{\frac{1}{N_{d}-1} \sum_{h \neq k} \sum_{j}\left[\frac{w_{C}^{D}}{N} \delta_{k} \delta_{h j}+w_{S}^{D} \delta_{h j}^{2}+\frac{w_{D}^{D}}{N-1} \sum_{n \neq j} \delta_{h n} \delta_{h j}\right]\right\}
\end{aligned}
$$

Since $\overline{\delta^{3}}=\overline{\delta \delta^{2}}=O\left(\delta^{4}\right)$,

$$
\begin{aligned}
\phi_{1} & =\frac{1}{N_{d}} \sum_{k} \frac{1}{N^{2}}\left\{\sum_{i} w_{k i}^{P} \delta_{k i}^{2}\right\}\left\{\sum_{h \neq k} \sum_{j} w_{k h j}^{D} \delta_{h j}\right\} \\
& =(1-m) \overline{\delta^{2}}\left\{w_{S}^{D} \overline{\delta^{2}}+w_{D}^{D} \overline{\delta \delta}\right\}
\end{aligned}
$$

By a similar calculation, we obtain

$$
\begin{aligned}
\phi_{2} & =\frac{1}{N_{d}} \sum_{k} \frac{1}{N^{2}}\left\{\sum_{i} w_{k i}^{P} \delta_{k i}\right\}\left\{\sum_{h \neq k} \sum_{j} w_{k h j}^{D} \delta_{h j}^{2}\right\} \\
& =\frac{1}{N_{d} N^{2}} \sum_{k}\left\{\sum_{i}\left[(1-m) \delta_{k i}+w_{S}^{P} \delta_{k i}^{2}+\frac{w_{D}^{P}}{N-1} \sum_{j \neq i} \delta_{k j} \delta_{k i}\right]\right\} \\
\{m & \left.m \overline{\delta^{2}}+\frac{1}{N_{d}-1} \sum_{h \neq k} \sum_{j}\left[\frac{w_{C}^{D}}{N} \delta_{k} \delta_{h j}^{2}+w_{S}^{D} \delta_{h j}^{3}+\frac{w_{D}^{D}}{N-1} \sum_{n \neq j} \delta_{h n} \delta_{h j}^{2}\right]\right\} \\
= & \frac{1}{N_{d} N^{2}} \sum_{k}(1-m) \delta_{k}\left[w_{C}^{D} \delta_{k} \overline{\delta^{2}}\right]+\frac{m \overline{\delta^{2}}}{N_{d} N} \sum_{k} \sum_{i}\left[w_{S}^{P} \delta_{k i}^{2}+\sum_{j \neq i} \frac{w_{D}^{P}}{N-1} \delta_{k j} \delta_{k i}\right] \\
= & \frac{(1-m)}{N^{2} N_{d}} w_{C}^{D} \sum_{k} \delta_{k}^{2}+m \overline{\delta^{2}}\left[w_{S}^{P} \overline{\delta^{2}}+w_{D}^{P} \overline{\delta \delta}\right]
\end{aligned}
$$

Using $\quad \sum_{k} \delta_{k}^{2}=\sum_{k}\left(\sum_{k} \delta_{k i}\right)^{2}=\sum_{k} \sum_{i} \sum_{j} \delta_{k i} \delta_{k j}=N_{d}\left(N(N-1) \overline{\delta \delta}+N \overline{\delta^{2}}\right)$, we have

$\phi_{2}=(1-m) \overline{\delta^{2}} w_{C}^{D}\left(\frac{N-1}{N} \overline{\delta \delta}+\frac{1}{N} \overline{\delta^{2}}\right)+m \overline{\delta^{2}}\left[w_{S}^{P} \overline{\delta^{2}}+w_{D}^{P} \overline{\delta \delta}\right]$

Since any individual in deme $h$ has a parent, the following equality always holds:

$\sum_{k \neq h} \sum_{i} \frac{w_{h k i}^{D}}{N}+\sum_{i} \frac{w_{h i}^{P}}{N}=1$

Thus,

$\sum_{k \neq h} \sum_{i} w_{h k i, h 1}^{D}+w_{h 1, h 1}^{P}+\sum_{i \neq 1} w_{h i, h 1}^{P}=w_{C}^{D}+w_{S}^{P}+w_{D}^{P}=0$,

and which also holds for any individual $j$ in deme $h$. Using this, we have

$\phi_{2}=-(1-m) \overline{\delta^{2}}\left(w_{S}^{P}+w_{D}^{P}\right)\left(\frac{N-1}{N} \overline{\delta \delta}+\frac{1}{N} \overline{\delta^{2}}\right)+m \overline{\delta^{2}}\left[w_{S}^{P} \overline{\delta^{2}}+w_{D}^{P} \overline{\delta \delta}\right]$

By a similar calculation, we have

$\phi_{3}=m \overline{\delta^{2}}\left(w_{S}^{D} \overline{\delta^{2}}+w_{D}^{D} \overline{\delta \delta}\right)$

Using $w_{S}=w_{S}^{P}+w_{S}^{D}, w_{D}=w_{D}^{P}+w_{D}^{D}$, and $w_{S}+R_{2} w_{D}=0$, we obtain

$\phi=-(1-m) \overline{\delta^{2}}\left[w_{D}^{P} \overline{\delta \delta}+w_{S}^{P} \overline{\delta^{2}}+\left(w_{S}^{P}+w_{D}^{P}\right)\left(\frac{N-1}{N} \overline{\delta \delta}+\frac{1}{N} \overline{\delta^{2}}\right)\right]$

By adding Eq. (B.26) and (B.43) and rewriting the moments in terms of relatedness coefficients, we finally obtain

$$
\begin{aligned}
& E\left[\overline{\delta \delta^{2}}{ }_{t+1} \mid \mathbf{z}_{t}=\mathbf{z}\right]=(1-m)^{2} \frac{N-1}{N} \overline{\delta \delta^{2}} \\
& \quad+4 V^{2} \frac{(1-m)}{N}\left(\left(2 R_{2}+(N-2) R_{3}\right) w_{D}^{P}+\left(1+(N-1) R_{2}\right) w_{S}^{P}\right)+O\left(\delta^{5}\right)
\end{aligned}
$$

Since only the first term in the above recursion for $\overline{\delta \delta^{2}}$ has a dominant order $O\left(\delta^{3}\right)$, we can approximate $\overline{\delta \delta^{2}}$ at the quasi-equilibrium given by

$\overline{\delta \delta^{2}}=4 V^{2}(1-m) \frac{\left(2 R_{2}+(N-2) R_{3}\right) w_{D}^{P}+\left(1+(N-1) R_{2}\right) w_{S}^{P}}{1+2 m(N-1)-m^{2}(N-1)}$

which can be simplified to

$\overline{\delta \delta^{2}}=4 V^{2} R_{2} \frac{\left(2 R_{2}+(N-2) R_{3}\right) w_{D}^{P}+\left(1+(N-1) R_{2}\right) w_{S}^{P}}{1-m}$.

By inserting Eq. (B.46) and $\overline{\delta^{3}}=0$ into Eq. (B.1), the effect on trait variance due to the perturbation of relatedness is then given by

$\Delta r=4 R_{2} \frac{\left(2 R_{2}+(N-2) R_{3}\right) w_{D}^{P}+\left(1+(N-1) R_{2}\right) w_{S}^{P}}{1-m} w_{D}$ 


\section{Appendix C}

The correspondence of $\Delta w$ to the first term of the 2nd-order derivative of invasion fitness $R_{m}^{\prime \prime}$ given in Eq. (18) in Ajar (2003) is clear, while the correspondence of $\Delta r$ to the second term is not trivial. Here we show this correspondence. The notations in Ajar (2003) can be translated into our notation as follows:

$F=\frac{1}{N}+\frac{N-1}{N} R_{2}$

$K=\frac{1}{N^{2}}+3 \frac{N-1}{N^{2}} R_{2}+\frac{(N-1)(N-2)}{N^{2}} R_{3}$

$\frac{\partial w_{p}}{\partial z_{\bullet}}=w_{S}^{P}-\frac{w_{D}^{P}}{N-1}, \quad \frac{\partial w_{p}}{\partial z_{0}}=\frac{N}{N-1} w_{D}^{P}, \quad \frac{\partial w}{\partial z_{0}}=\frac{N}{N-1} w_{D}$

$w_{p}=1-m$

Then after some calculations our result can be rewritten as

$\Delta r=4 F(N-1)\left(K \frac{\partial w_{p}}{\partial z_{0}}+F \frac{\partial w_{p}}{\partial z_{\bullet}}\right) w_{p} \frac{\partial w_{p}}{\partial z_{0}}$

which is identical to the second term of Eq. (18) in Ajar (2003) except a factor $1 / d$. This factor also appears in the first term corresponding to $\Delta w$, and thus our condition reduces to the condition given by Ajar (2003).

We also note the correspondence to the results of Day (2001), which shows the ESS condition only for a case $N=2$ and hence $R_{3}$ does not exist. The translation of variables is given by

$w_{S S}=\frac{\partial^{2} G}{\partial x^{2}}, \quad w_{S D}=\frac{\partial^{2} G}{\partial x \partial y}, \quad w_{D D}=\frac{\partial^{2} G}{\partial y^{2}}, \quad w_{D}=\frac{\partial G}{\partial y}$

$\rho_{2}=R_{2}$

and then Eq. (D.5) in Day (2001) has exactly the same term as our $\Delta w$ plus the additional term

$2 \frac{d \rho_{2}}{d x} \frac{\partial G}{\partial y}$

which clearly corresponds to our $\Delta r$ as both of them capture the effect of the perturbation of relatedness.

\section{Appendix D}

Fitness is defined to be the expected number of successful offspring. In terms of fecundity $F_{k i}=F_{k i}(\mathbf{z})$, which is determined by the pairwise payoff function $f\left(z_{i}, z_{j}\right)$ as in Eq. (33), the fitness function is obtained as follows. The total amount of gametes in deme $k$ is given by

$N \times F_{k}=(1-m) \sum_{j=1}^{N} F_{k j}+\frac{m}{N_{d}-1} \sum_{l=1, l \neq k j=1}^{N_{d}} \sum_{l j}^{N} F_{l j}$

where $F_{k}$ is the average fecundity in deme $k$. Then the fitness function is given by

$$
\begin{aligned}
w_{k i} & =w_{k i}^{P}+w_{k i}^{D} \\
& =\frac{(1-m) F_{k i}}{F_{k}}+\sum_{l=1, l \neq k}^{N_{d}} \frac{m /\left(N_{d}-1\right) F_{k i}}{F_{l}}
\end{aligned}
$$

It is straightforward to confirm that the fitness function is normalized as $\bar{w}=1$ and symmetric as $w_{k i}(\overline{\mathbf{z}})=1$ holds.

The fitness derivatives appearing in our branching condition can be derived by differentiating Eq. (D.2). In the infinite-deme limit $\left(N_{d} \rightarrow \infty\right)$, we have

$w_{S S}=\frac{1}{F}\left(F_{k i, k i k i}-(1-m)^{2} F_{k, k i k i}\right)+\frac{2(1-m)^{2}}{F^{2}}\left[(1-m)\left(F_{k, k i}\right)^{2}-F_{k i, k i} F_{k, k i}\right]$ where $F$ is fecundity evaluated at the trait distribution $\left(z^{*}, z^{*}, \ldots\right.$, $\left.z^{*}\right)$. Similarly, we obtain

$$
\begin{aligned}
& w_{D D}=(N-1) \\
& \quad \times\left[\frac{1}{F}\left(F_{k i, k j k j}-(1-m)^{2} F_{k, k j k j}\right)+\frac{2(1-m)^{2}}{F^{2}}\left[(1-m)\left(F_{k, k j}\right)^{2}-F_{k i, k j} F_{k, k j}\right]\right]
\end{aligned}
$$

$$
\begin{aligned}
w_{S D}= & (N-1) \\
\times & {\left[\begin{array}{l}
\frac{1}{F}\left(F_{k i, k i k j}-(1-m)^{2} F_{k, k i k j}\right) \\
+\frac{(1-m)^{2}}{F^{2}}\left[2(1-m) F_{k, k i} F_{k, k j}-F_{k i, k j} F_{k, k i}-F_{k i, k i} F_{k, k j}\right]
\end{array}\right] } \\
w_{D D^{\prime}}= & (N-1)(N-2) \\
& \times\left[\begin{array}{l}
\frac{1}{F}\left(F_{k i, k j k r}-(1-m)^{2} F_{k, k j k r}\right) \\
+\frac{2(1-m)^{2}}{F^{2}}\left[(1-m)\left(F_{k, k j}\right)^{2}-F_{k i, k j} F_{k, k j}\right]
\end{array}\right]
\end{aligned}
$$

where $i, j$, and $r$ are different from each other. Fecundity derivatives are explicitly given by pairwise payoff function $f$ as follows:

$F_{k i, k i}=f_{1}, \quad F_{k i, k i k i}=f_{11}$

$F_{k, k i}=F_{k, k j}=\frac{1}{N}\left(f_{1}+f_{2}\right)$

$F_{k, k i k i}=F_{k, k j k j}=\frac{1}{N}\left(f_{11}+f_{22}\right)$

$F_{k i, k j}=f_{2} /(N-1), \quad F_{k i, k j k j}=f_{22} /(N-1)$

$F_{k i, k i k j}=f_{12} /(N-1), \quad F_{k, k i k j}=F_{k, k j k r}=(2 / N(N-1)) f_{12}, \quad F_{k i, k j k r}=0$

where a subscript to $f$ means a variable with respect to which a partial derivative is calculated, e.g., $f_{12}=\partial^{2} f\left(z_{1}, z_{2}\right) / \partial z_{1} \partial z_{2}$ (Wakano and Lehmann, 2012). Rewriting fitness derivatives (Eqs. (D.3)-(D.6)) in terms of payoff derivatives, then putting them to Eq. (27), we have the explicit form of $\Delta w$ in terms of payoff derivatives. Similar process can be applied for $\Delta r$ and hence $Q_{E S}$. The result is not shown here since it is very lengthy. This can be applied to any kind of pairwise payoff function $f\left(z_{1}, z_{2}\right)$. To derive the condition for our example case, putting an explicit form of payoff function (Eqs. (34)) into this result and solving Eqs. (13) and (23) to replace $R_{2}$ and $R_{3}$ as functions of $m$ and $N$, we obtain an explicit expression of $Q_{E S}$ by Mathematica, which is too cumbersome to be presented here.

Note that $w_{S S}, w_{S D}, w_{D D}, w_{D D^{\prime}}$ as well as $R_{2}, R_{3}$ depend on $m$ and $N$. Even in the Wright-Fisher updating, $m$ and $N$ do not vanish as they do in a case of the mean trait dynamics. Thus, the resultant condition is a very complicated function of $m$ and $N$. Weak fecundity selection assumed in Eq. (37) removes many of these complexities.

\section{References}

Abrams, P.A., Matsuda, H., Harada, Y., 1993. Evolutionarily unstable fitness maxima and stable fitness minima of continuous traits. Evol. Ecol. 7, 465-487.

Ajar, E, 2003. Analysis of disruptive selection in subdivided populations. BMC Evol Biol. 3, 22.

Brännström, A., Gross, T., Blasius, B., Dieckmann, U., 2011. Consequences of fluctuating group size for the evolution of cooperation. J. Math. Biol. 63, 263-281.

Bulmer, M.G., 1986. Sex-ratio in geographically structured populations. Heredity 56 69-70.

Champagnat, N., Ferriere, R., Meleard, S., 2006a. Unifying evolutionary dynamics: from individual stochastic processes to macroscopic models. Theor. Popul. Biol. 69, 297-321.

Champagnat, N., Ferriere, R., Meleard, S., 2006b. Unifying evolutionary dynamics: From individual stochastic processes to macroscopic models. Theor. Popul. Biol. 69, 297-321.

Day, T., 2001. Population structure inhibits evolutionary diversification under competition for resources. Genetica 112-113, 71-86.

Day, T., Taylor, P.D., 1996. Evolutionarily stable versus fitness maximizing life histories under frequency-dependent selections. Proc. Roy. Soc. B 263, 333-338.

Dieckmann, U., Law, R., 1996. The dynamical theory of coevolution: a derivation from stochastic ecological processes. J. Math. Biol. 34, 579-612. 
Doebeli, M., Hauert, C., Killingback, T., 2004. The evolutionary origin of cooperators and defectors. Science 306, 859-862.

Eshel, I., 1983. Evolutionary and continuous stability. J. Theor. Biol. 103, 99-111.

Frank, S.A., 1998. Foundations of Social Evolution. Princeton University Press, Princeton.

Geritz, S.A.H., Metz, J.A.J., Kisdi, E., Meszena, G., 1997. Dynamics of adaptation and evolutionary branching. Phys. Rev. Lett. 78, 2024-2027.

Geritz, S.A.H., Kisdi, E., Meszena, G., Metz, J.A.J., 1998. Evolutionarily singular strategies and the adaptive growth and branching of the evolutionary tree. Evol. Ecol. 12, 35-57.

Guillaume, F., Perrin, N., 2006. Joint evolution of dispersal and inbreeding load. Genetics 173, 497-509.

Hamilton, W.D., 1964. The genetical evolution of social behavior I. J. Theor. Biol. 7 $1-16$.

Iwasa, Y., Pomiankowski, A., Nee, S., 1991. The evolution of costly mate preferences II. The 'handicap' principle. Evolution 45, 1431-1442.

Jabin, P., Raoul, G., 2011. On selection dynamics for competitive interactions. J. Math. Biol. 63, 493-517.

Kimura, M., 1965. A stochastic model concerning the maintenance of genetic variability in quantitative characters. Proc. Nat. Acad. Sci. U.S.A. 54, 731-736.

Lehmann, L., 2008. The adaptive dynamics of niche constructing traits in spatially subdivided populations: evolving posthumous extended phenotypes. Evolution 62, 549-566.

Lehmann, L., 2012. The stationary distribution of a continuously varying strategy in a class-structured population under mutation-selection-drift balance. J. Evol. Biol. 25, 770-787.

Lehmann, L., Rousset, F., 2010. How life history and demography promote or inhibit the evolution of helping behaviours. Philos. Trans. Roy. Soc. B 365, 2599-2617.

Leturque, H., Rousset, F., 2002. Dispersal, kin competition, and the ideal free distribution in a spatially heterogeneous population. Theor. Popul. Biol. 62, 169-180.

Metz, J.A.J., Gyllenberg, M., 2001. How should we define fitness in structured metapopulation models? Including an application to the calculation of evolutionary stable dispersal strategies. Proc. Roy. Soc. B 268, 499-508.

Metz, J.A.J., Nisbet, R.M., Geritz, S.A.H., 1992. How should we define "fitness" for general ecological scenarios? Trends Ecol. Evol. 7, 198-202.
Metz, J.A.J., Geritz, S.A.H., Meszena, G., Jacobs, F.J.A., van Heerwaarden, J.S., 1996. Adaptive dynamics, a geometrical study of the consequences of nearly faithful reproduction. In: van Strien, S.J., Lunel, S.M. Verduyn (Eds.), Stochastic and Spatial Structures of Dynamical Systems. A'dam, North-Holland, pp. 183-231.

Mirrahimi, S., Perthame, B., Wakano, J.Y., 2012. Evolution of species trait through resource competition. J. Math. Biol. 64, 1189-1223.

Parvinen, K., Metz, J.A.J., 2008. A novel fitness proxy in structured locally finite metapopulations with diploid genetics, with an application to dispersal evolution. Theor. Popul. Biol. 73, 517-528.

Pen, I., 2000. Reproductive effort in viscous populations. Evolution 54, 293-297.

Queller, D.C., 1994. Genetic relatedness in viscous populations. Evol. Ecol. 8, 70-73.

Rousset, F., 2004. Genetic Structure and Selection in Subdivided Populations. Princeton University Press, Princeton.

Rousset, F., Ronce, O., 2004. Inclusive fitness for traits affecting metapopulation demography. Theor. Popul. Biol. 65, 127-141.

Roze, D., Rousset, F., 2003. Selection and drift in subdivided populations: a straightforward method for the deriving diffusion approximations and applications involving dominance, selfing, and local extinctions. Genetics 165, 2153-2166.

Sasaki, A., Dieckmann, U., 2011. Oligomorphic dynamics for analyzing the quantitative genetics of adaptive speciation. J. Math. Biol. 63, 601-635.

Sasaki, A., Ellner, S., 1995. The evolutionarily stable phenotype distribution in a random environment. Evolution 49, 337-350.

Taylor, P.D., 1988. An inclusive fitness model for dispersal of offspring. J. Theor. Biol. 130, 363-378.

Wakano, J.Y., Iwasa, Y., 2013. Evolutionary branching in a finite population: deterministic branching versus stochastic branching. Genetics 193, 229-241.

Wakano, J.Y., Lehmann, L., 2012. Evolutionary and convergence stability for continuous phenotypes in finite populations derived from two-allele models. J. Theor. Biol. 310, 206-215.

Wenseleers, T., Gardner, A., Foster, K.R., 2010. Social evolution theory: a review of methods and approaches. In: Szekely, T., Moore, A., Komdeur, J. (Eds.), Social Behaviour: Genes, Ecology and Evolution. Cambridge University Press, pp. 132-158. 